\title{
Expert Consensus on Standardized Practice of Oral Contrast Agent-Enhanced Gastric Ultrasonography (Shanghai, 2020 edition)
}

\author{
Gastrointestinal Ultrasound Task Force, Ultrasound Research and Education Institute, Clinical Research Center \\ of Interventional Medicine, School of Medicine, Tongji University; Shanghai Engineering Research Center of
} Ultrasound Diagnosis and Treatment; National Clinical Research Center of Interventional Medicine; Abdominal Group, Ultrasound Branch, Shanghai Medical Association; Abdominal and Vascular Ultrasound Sub-Committee, Society of Ultrasound Medicine, Shanghai Association of Social Medical Institutions

Received April 27, 2021; revision received May 14, 2021; accepted May 16, 2021

Advanced Ultrasound in Diagnosis and Therapy 2021;02:115-133

DOI: 10.37015/AUDT.2021.210009

$\mathrm{T}$ he research and application of oral contrastenhanced in gastric ultrasonography (hereinafter referred to as: gastric ultrasonography) provides an alternative imaging modality to gastroscope, endoscopic ultrasound and computed tomography (CT) in the diagnosis and treatment of gastric lesions when those modalities are not accessible [1]. Gastric ultrasonography is a combination of oral acoustic contrast agent and ultrasound imaging technology. Conventional transabdominal ultrasonography could display normal gastric wall structure, as well as the location, size, shape of the lesion, and its relation with the gastric wall after contrast agents filling the gastric cavity. Color Doppler flow imaging (CDFI) and intravenous contrast-enhanced ultrasound can detect the blood perfusion of the gastric lesions as well. Therefore, gastric ultrasonography effectively provides non-invasive and visual diagnostic information to clinicians [2-5]. Despite this modality has become more and more important in clinical diagnosis and treatment of gastric diseases [6,7], there is still lack of unified and standardized operation guidiline.

The present consensus focused on the pre-procedural preparation of gastric ultrasonography, equipment parameter setting, contrast preparation requirements, scanning methods and ultrasonic imaging sections, to provide preliminary suggestions and guidance for the standardized practice of gastric ultrasonography. This consensus was established for academic experience exchange and reference only, and should be updated and supplemented with the advancement of ultrasonic technology.

\section{Levels of Evidence and Recommendations}

This consensus attempted to give recommendations based on the corresponding levels of evidence (Table 1) mainly from comprehensive literature analysis, relevant clinical studies, and the experts' experiences in the compilation group; so as to fully highlight the scientific concept of evidence-based medicine.

\section{History and the Current State of Gastric Ultrasonography}

In the late 1950s, A-mode ultrasound was applied in patients with water-filled empty stomach, which initiated the era of clinical ultrasonic application in the diagnosis of gastric diseases [3]. However, the A-mode gastric ultrasonography is greatly limited due to the interference of gastric gas and contents.

\footnotetext{
* Corresponding authors: Huixiong Xu, Tenth People's Hospital Affiliated to Tongji University, Shanghai, China.e-mail: xuhuixiong@126. com; Lianfang Du, First People's Hospital Affiliated to Shanghai Jiao Tong University School of Medicine, Shanghai, China. e-mail: du_ lf@163.com; Li Shen, Chongming Branch of Xinhua Hospital Affiliated to Shanghai Jiao Tong University School of Medicine, Shanghai, China.e-mail:shenlicm@126.com unrestricted use, distribution and reproduction in any medium provided that the original work is properly attributed.
} 
Table 1 Evidence level and recommendation category

\begin{tabular}{ll}
\hline Level of evidence & Category of Recommendation \\
\hline A & Strong recommendation: Evidence-based evidence is certain, improve the clinical application, benefits outweigh the disadvantages. \\
B & Recommendation: Evidence-based evidence is good, improve the clinical application effect, benefits outweigh the disadvantages. \\
C & Neutral: Insufficient evidence, lack of evidence, or contradictory results, and the pros and cons cannot be assessed. \\
D & No recommendation: Based on expert opinion. \\
\hline E &
\end{tabular}

With the development of B-mode ultrasound in the 1970s, gastric ultrasonography became a hotspot in clinical application and research. Under B-mode ultrasound, normal gastric wall sonogram presents five layers of different echo intensity, which roughly correspond to the mucosal layer, mucosal muscular layer, submucosal layer, muscularis propria, serosa layer and loose fat tissue observed under microscope (Fig. 1) [4]. Studies have demonstrated that water-soluble gastric cavity filling agents were helpful in ultrasonic imaging and found that gastric neoplasms resemble renal sections on sonograms. Accordingly, the concept of "false kidney sign" of gastric tumor was described $[8,9]$.
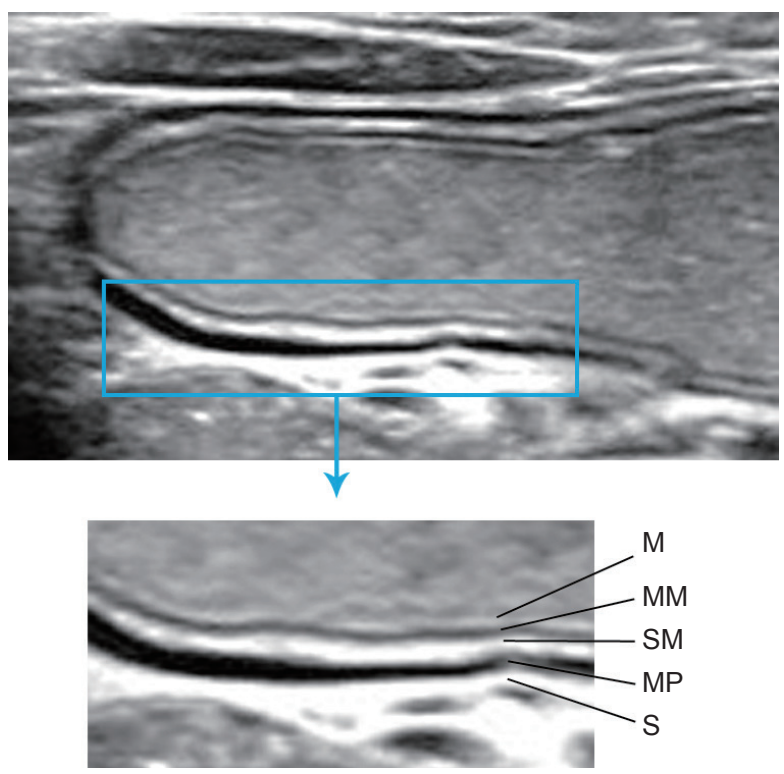

Figure 1 Ultrasound image of normal gastric wall. M, the first layer of hyperecho, equivalent to the echo of the gastric cavity interface and the superficial layer of mucosa; MM, the second layer is hypoechoic, equivalent to the mucosal muscular layer; SM, the third layer of hyperecho, equivalent to the submucosa; MP, the fourth layer is hypoechoic, equivalent to muscularis propria; $\mathrm{S}$, the fifth layer is hyperechoic, equivalent to the serosal layer and extrasosal adipose tissue echo.

In the late 1980s, the advancement in gastric cavity filling agents promoted the application and researches of gastric ultrasonography [10-12]. Guo et. al. [13] developed a new generation of food-type "echoic contrast agent". Also, it was proposed that cellulose gastric cavity filling agent can significantly reduce the interference of gas and improve the image quality of gastric wall compared with water [14]. These studies established the foundation for the development of gastric ultrasonography [15].

Since 21 st century, the gastric cavity filling agent had been gradually replaced by "gastric cavity contrast agent", with wide clinical application of ultrasonic contrast agent such as SonoVue. Meanwhile, the focus of gastric ultrasonography studies shifted to the following aspects: (1) dual contrast-enhanced ultrasound (CEUS), i.e., oral and intravenous combined with CEUS for the diagnosis and treatment of gastric cancer[16,17]; (2) primary ultrasonographic screening of gastric cancer in healthy population; (3) development of gastric ultrasonic image report and data system (SU-RADS)[18];(4) standardization of gastric ultrasonography examination technology; (5) functional tests of the stomach, such as diagnosis of gastroesophageal reflux disease.

\section{Indications and Contraindications [19-23]}

\subsection{Indications}

3.1.1 Patients with symptoms and signs of upper digestive tract diseases, such as suspected gastric neoplastic lesions, gastric ulcers, gastric foreign materials, esophageal and gastric varices, and further investigations of other gastric lesions.

3.1.2 Identify whether the mass is stomach originated, examine the relationship between the mass and the stomach wall, and/or the invasion of surrounding tissues and organs such as liver or pancreas.

3.1.3 Patients requiring follow-up for gastric lesions, such as peptic ulcer and gastric cancer, observation and evaluation of efficacy of neoadjuvant chemotherapy.

3.1.4 Stomach function tests, such as stomach receptivity, emptiness and gastroesophageal reflux diseases.

3.1.5 Patients intolerant to gastroscopy or endoscopic ultrasound.

3.1.6 Preliminary screening in people of high risk of gastric cancer to improve early diagnosis and promote 
early treatment.

Expert opinion: Gastric ultrasonography provide an efficient imaging tool without allergic and other toxic side effects in clinical practice, which can be used as an important supplement to gastroscopy. It is sensitive to gastric mucosal, submucosal, and extragastric lesions, and also could detect perigastric lymph node enlargement.

\section{Strength of Recommendation: A.}

\subsection{Contraindications}

3.2.1 Patients who under order of "nothing by mouth," such as severe gastric retention, perforation of gastric or duodenal ulcer, emergency cases of gastrointestinal trauma.

3.2.2 Uncooperative examinee due to severe mental illness, cognitive impairment, dysphagia, persistent hiccups or physical exhaustion.

3.2.3 Patients with severe obesity, deep or high gastric position, and intestinal gas significantly interfering scanning, etc.

3.2.4 Require explicit exclusion of gastric tumor.

Expert Opinion: The aforementioned items elaborated the situations precluding applications of gastric ultrasonography, as well as the potential limitations that are still presently unknown, which should be assessed in further studies.

\section{Strength of recommendation: E.}

\section{Optimization of Ultrasonic Equipment and Imaging Parameters [24-25]}

\subsection{Ultrasonic equipment}

4.1.1 Equipment selection: portable or desktop abdominal ultrasound diagnostic equipment according to the examination scenario and hospital equipment configuration.

4.1.2 Probe and frequency: (1) conventional abdominal convex array probe, the frequency of $1.0-6.0 \mathrm{MHz}$ probe is preferred, which has the wide scanning field, deep penetration and high resolution; (2) for children, thin people, and the lesion close to the anterior abdominal wall, the high frequency linear array probe with a frequency of $4.0 \sim 12.0 \mathrm{MHz}$ is preferred, which has high spatial resolution of tissue to enhance the gastric wall or lesion display.

4.1.3 Others: Harmonic imaging, transvenous contrastenhanced ultrasound, three-dimensional ultrasound, shear-wave elastic ultrasound and other new technologies could be used in differential diagnosis of gastric lesions.

\subsection{Settings and optimization of imaging parameters}

Optimal setting and adjustment of parameters could improve the quality of gastric imaging. If possible, various imaging settings could be saved for different conditions, such as obesity, adults and children.

4.2.1 Probe frequency: frequency determines the penetration and resolution. The depth of the gastric wall to the abdominal wall, and the thickness of the abdominal wall varied individually. Therefore, the optimal ultrasound imaging should be considered comprehensively. Under the premise of ensuring ultrasonic penetration, high frequency ultrasonic probe should be selected as much as possible.

4.2.2 Gain and time-gain compensation: different gastric contrast agents have different acoustic attenuation coefficients. Among them, water filling agent is most likely to enhance the echo of the posterior wall of the stomach significantly, which reduce the identification of the hierarchical structure of the gastric wall. When this happens, the total gain or the far field gain compensation should be reduced appropriately.

4.2.3 Depth: Adjust the display depth can zoom in the ultrasonogram. Zooming in on ultrasonogram can help to observe the microstructure and morphology of the gastric wall, and increase the frame rate to improve the time-effectiveness and continuity. The recommended maximum imaging depth is about $100 \sim 120 \mathrm{~mm}$.

4.2.4 Dynamic range: The gastric wall has a rich hierarchical structure, and each layer is relatively thin. Although increasing the dynamic range can enrich the echo signals, it may decrease the resolution. Appropriate compression of dynamic range can improve the contrast and resolution of gastric wall hierarchical structure. The recommended dynamic range is about $60 \sim 70 \mathrm{~dB}$, which should be adjusted according to the equipment.

4.2.5 Imaging frame rate: It is common to observe the stomach under peristalsis. Therefore, it is necessary to increase the frame rate and presets to improve observation of the structure in motion. (1) Focus points: reduce the number of focus points to improve the frame rate. No more than two is recommended, and one point is preferred. (2) Image overlay processing: reduce image overlay processing to improve the real-time performance, and adjust according to the specific equipment. (3) Line density: the higher the line density, the better the image. However, increasing the linear density will decrease the frame rate. In order to ensure the optimal frame rate, the image line density should be reduced appropriately, and the highest line density is not recommended.

4.2.6 Others: Adjusting the local magnification function, edge enhancement and other imaging techniques appropriately provides better display of the gastric wall structure and lesions.

Expert Opinion: The above items explained the importance and appropriately adjusted settings and parameters of ultrasonic equipment in optimizing clinical 
application of gastric ultrasonography.

Strength of Recommendation: B.

\section{Commonly Used Contrast Agents and Their}

\section{Preparation and Application $[3,19,23]$}

The oral contrast agent is mainly used to fill the gastric cavity and eliminate or reduce the interference of ultrasonic transmission from gastric gas, food residue and mucus. The preparation and application of contrast agent directly affect the quality of gastric ultrasonic imaging.

\subsection{Commonly used contrast agents}

5.1.1 Anechoic contrast agent: Plain water, mineral water or traditional Chinese medicine preparations (such as gastric ultrasound synergistic solution) etc. are commonly used. After oral administration, the filling of the gastric cavity shows an anechoic area, which is conducive to the display and detection of hyperechoic lesions in the gastric mucosa. However, it is possible to miss hypoechoic lesions in gastric mucosa.

Expert opinion: Anechoic contrast agent, especially plain water, is practicable and effective. Which could be used as a first-line contrast agent in gastric ultrasonography.

Strength of Recommendation: B.

\subsubsection{Echoic contrast agent:}

\subsubsection{Gastric ultrasonography contrast agents:} Several agents were approved by the Chinese FDA for commercial use, such as "stomach window acoustic contrast agent" and "gastric ultrasonography aid agent." They are also named "food-based contrast agent" as they mainly consist of grain powder, which could mitigate echoic interference from gastric cavity gas and mucus with the advantage of long stomach retention time and no toxic side effects. After oral administration, the filling of the gastric cavity would show a hyperechoic area, which is helpful to the display and detection of hypoechoic lesions in the gastric mucosa. However, it is possible to miss gastric mucosal hyperechoic lesions, such as gastric polyps.

Expert opinion: The commercially available gastric ultrasonography contrast agents, such as "gastric window acoustic contrast agent" (Xin-Zhang ${ }^{\circledR}$, Huqingyutang Pharmaceuticals Co., Hangzhou, China), are originated in China with independent intellectual property patent, they have been proven to be stable and reliable in clinical practice, and can be used as first-line contrast agents for gastric ultrasonography.

Strength of Recommendation: A.

5.1.2.2 Microbubbles ultrasound contrast agent: The most commonly used agent is Sonovue (Bracco, Italy), which is phospholipid-coated sulfur hexafluoride (SF6) Microbubble contrast agent, mainly used for intravenous contrast ultrasound. It also can be used orally for gastric ultrasonography after appropriate dilution and modulation. The advantages of this agent are that it enhances the gastric cavity filling significantly, provides high resolution of gastric cavity and gastric wall mucosa, which facilitates the evaluation of gastric cavity stenosis and gastric anastomotic fistula.

Expert opinion: Oral microbubbles contrast agent should be examined in the harmonic imaging mode of contrast-enhanced ultrasound. Due to the decreased resolution of two-dimensional ultrasound, which negatively affects the display and observation of gastric wall hierarchy, it is not recommended as a first-line contrast agent.

Strength of Recommendation: $C$.

5.1.2.3 Others: lotus root powder, starch and sesame paste, etc., are generally not recommended due to the unstable or unsatisfactory imaging effect.

Expert opinion: Ultrasonography is unreliable with gastric contrast of lotus root powder, starch, or sesame paste; therefore, they are not recommended for routine application.

\section{Strength of Recommendation: D.}

\subsection{Contrast agent preparation}

5.2.1 Anechoic contrast agent

5.2.1.1 Preparation: there is no special preparation for plain water, mineral water, etc.;

5.2.1.2 Notes: Gas free or degassed mineral water is preferred in order to reduce gas interference.

\subsubsection{Echoic contrast agent}

\subsubsection{Gastric ultrasound contrast agent}

Preparation: Contrast agent was prepared strictly according to product instructions. Appropriate water temperature combined with sufficient mixing is the key. A qualified contrast agent is in a status of suspension, which is uniform without obvious precipitation and has certain viscosity;

Precautions: Check temperature of contrast agent before oral administration. It should be stirred again before use if sedimented. According to the existing experience, the efficiency and quality of electric stirrers may be better than manual stirring.

\subsubsection{Microbubble ultrasound contrast agent:}

Preparation: According to the preparation instructions of SonoVue contrast agent $(4.8 \mathrm{mg} / 5 \mathrm{ml}$, Bracco, Italy), $5 \mathrm{ml}$ saline injected then shake thoroughly until the lyophilized powder completely dissolves into milky white suspension. Afterwards, extract $0.5 \mathrm{ml}$ suspension, added into $500 \sim 700 \mathrm{ml}$ plain, pure or mineral water. Shake well for use $[17,26]$. 
Precautions: the preparation shall comply with the instructions, relevant specifications or guidelines for the use of microbubble ultrasonic contrast agent.

Expert opinion: The accurate and standardized preparation of contrast agent is directly related to the effectiveness of ultrasound diagnosis. Inspectors must pay high attention to ensure the preparation meets the diagnostic requirements.

\section{Strength of Recommendation: A.}

\subsection{Application of contrast agent}

\subsubsection{Dosage}

Based on the requirements of standardization of gastric ultrasonography [1], it is necessary to set a standard reference for oral dose. At moderate fullness, the stomach volume of an adult is about $1500 \mathrm{ml}$ [27]. Because the size and capacity of the stomach are different, the specific dosage should be adjusted individually. (1) For adults, the dosage is generally 500 $1000 \mathrm{ml}$, and the recommended dosage is about $700 \mathrm{ml}$.

(2) Recommended approximate dosage for children: $10 \mathrm{ml}$ for newborns, $100 \sim 200 \mathrm{ml}$ for $1 \sim 3$ years old, 200 $\sim 400 \mathrm{ml}$ for $3 \sim 10$ years old, $400-600 \mathrm{ml}$ for $10 \sim 15$ years old and above. (3) Oral dose can be added until the satisfactory filling of the gastric cavity [28].

Expert Opinion: Gastric ultrasonography is based on filling the gastric cavity essentially. Insufficient filling may result in invalid or failed scanning, while excessive filling may affect the accuracy. Therefore, the examiner should grasp the accurate amount, and guide the patient to take adequate contrast agent.

\section{Strength of Recommendation: A.}

\subsubsection{Oral administration}

Before scanning, patients should take the contrast agent continuously and as quickly as possible, but be careful to avoid choking. If not, gas may interfere the ultrasonic imaging. In the diagnosis of functional or organic diseases such as achalasia of cardia, cardiac space-occupying lesions, varices of esophagus and stomach fundus, as well as gastric cavity failed to fill normally after taking enough contrast agent, sonographer should carry out the scan while exmaniee taking the contrast agent simultaneously to observe real-time imaging of contrast agent passing through cardia and filling gastric cavity.

\subsubsection{Precautions}

5.3.3.1 Anechoic ultrasound contrast agent: Preferred for infants, elderly, and other weak physique, or suspected hyperechoic gastric lesions, such as gastric polyps and gastric stones. Do not scan immediately after drinking water. It is necessary to wait for about 5 minutes until the water is still in the gastric cavity, and gas bubbles mixed with water float up and dissipate, scan should not procced until the gastric cavity is shown anechoic [28].

5.3.3.2 Echoic contrast agents: Obtain history of drug allergy, and allergy to the contrast agent ingredients. Sonovue should not be mixed with other medications. When necessary, double gastric contrast examination combining oral and intravenous contrasts can be performed to obtain diagnostic information such as gastric lesions and characteristics of vasculatures in surrounding tissues [17,29-30].

Expert Opinion: Examiners should accurately grasp the application of contrast agents and relevant precautions, to ensure the quality and safety of scanning.

\section{Strength of Recommendation: B.}

\section{Preparation Before Inspection [19-21,23,31]}

\subsection{Patient preparations}

6.1.1 Prior to the examination, avoid spicy, fried, or grilled food, and any other strong irritant or indigestible food. Also restrain from beans, sweet potatoes and other gaseous food. Alcohol is prohibited.

6.1.2 Fasting on the examination day, adults for $6 \sim 8 \mathrm{~h}$ (for hypertension, heart disease and diabetes patients, a small amount of water is acceptable for oral routine medication), infants for $3 \mathrm{~h}$. Smoking is prohibited before the examination. Also, less speaking to reduce the gas swallowed if possible.

6.1.3 X-ray barium or gastroscopy can interfere with ultrasound scanning because of Barium stranded or gas accumulation in the gastric cavity. So, it should be performed at least $2 \mathrm{~d}$ after those examinations.

6.1.4 Fasting on patients with pyloric obstruction depends on their condition. Generally, fasting should be 2 $\sim 3$ days; if necessary, examination should be performed after gastric lavage.

6.1.5 Medication is unnecessary. For hyperperistalsis patients, muscle spasmodic can be administrated 15 minutes before examination, such as $0.5 \mathrm{mg}$ atropine or $10 \mathrm{mg}$ scopolamine.

\subsection{Sonographer preparations}

6.2.1 Sonographer should be familiar with the indications and contraindications of examination, as well as examinee's preparation. If the examinee did not fast as require, the examination should be rescheduled.

6.2.2 Acquire the examinee's history, purpose and requirements of examination, as well as the results of gastroscopy, $\mathrm{CT}$ and other examinations.

6.2.3 Examination should be scheduled in the early morning to shorten the waiting time as much as possible to prevent the patient from swallowing more gas due to talking or other reasons. 
6.2.4 Introduce the cooperation matters to the examinee briefly, such as the purpose of the contrast agent and posture requirements, so as to help patients relaxed.

6.2.5 Complete the contrast agent preparation in strict accordance with the instructions for use.

Expert Opinion: The above items emphasized the importance and necessity of preparation before examination, and pointed out the importance for both examinees and sonographers to be prepared before examination.

Strength of Recommendation: B.

\section{Examination Process, Position and Scanning Protocol}

\subsection{Examination process}

7.1.1 History inquiry: Focus on the symptoms and signs of the examinee's digestive system, and other recent imaging results such as gastroscopy.

7.1.2 Fasting evaluation: Before gastric filling, fasting gastric ultrasonography can be performed preliminarily to evaluate whether there are abnormalities in the stomach, duodenum and adjacent organs, such as spaceoccupying lesions and gastric retention.

7.1.3 Scanning sequency: After oral contrast agent, gastric ultrasonography was completed in a certain order. The basic examination order is: cardia and fundus of the stomach $\rightarrow$ middle upper and lower part of the stomach $\rightarrow$ gastric angle $\rightarrow$ gastric antrum $\rightarrow$ pylorus and duodenal sphere, descending and horizontal part $\rightarrow$ cardia and fundus of the stomach, achieving a "closed loop" of gastric scanning.

7.1.4 Comprehensive evaluation: complete the detection of the stomach, including the surface projection, adjacent relationship, the tissue structure of the gastric wall and the morphological characteristics of lesions, etc.

7.1.5 Key detection :(1) Patient with careful scanning should be carried out in common lesion sites (cardia, gastric angle and gastric antrum, etc.), difficult imaging sites (cardia, gastric fundus and pylorus), and difficult imaging cases (such as severe obesity, history of upper abdominal operation and spinal deformity, etc.). (2) Once gastric lesions are found, the location, size range, morphological characteristics, origin location, and depth of infiltration should be evaluated comprehensively, and the perfusion should be detected by color Doppler ultrasound.

7.1.6 Record and report: Document what you see in certain order (recommended report seen in Appendix 1).

7.1.7 Store all images related to diagnosis and differential diagnoses, including recording static and cine data.
Expert Opinion: The report document emphasizes the importance and necessity of standardizing the gastric ultrasonography process, including the core requirements of obtaining medical history, sequential examination, and focus observation.

\section{Strength of Recommendation: A.}

\subsection{Scanning position [19,21,32-33]}

7.2.1 Common position: (1) Standing position: It is often used to examine the distal stomach, such as the middle and lower stomach, stomach angle, gastric antrum, pylorus and the duodenal bulb, and diagnose gastric ptosis. Usually, the patient is placed in the left anterior position to scan cardia and fundus of the stomach. Next, facing the sonographer to scan the gastric horn, gastric body and gastric antrum. Finally, the antrum, pylorus, and duodenum are scanned in the right anterior position. (2) Sitting position: mainly used for examinee whom is intolerant to standing position. (3) Supine position: it is often used to scan the proximal stomach, such as the cardia, fundus, and the upper stomach.

7.2.2 Supplementary position: (1) Right $45^{\circ}$ lateral position is usually used for unsatisfactory imaging of gastric antrum, gastric horn and duodenal bulb. In addition, in the right decubitus position, the position of the cardia moves downward, which is conducive to the scanning of the xiphoid process, and can be used as a supplementary examination position for patients with cardiac imaging difficulties. (2) Left $45^{\circ}$ lateral position is often used when gastric fundus and cardia imaging is not satisfactory. (3) Others.

Expert opinion: Because of substances and gravity, the gas and mucus in the gastric cavity often gather in the upper gastric cavity, while the contrast agent is concentrated in the lower gastric cavity. When position is changed, the highest and lowest part of the gastric cavity will change accordingly. Therefore, the appropriate position should be selected according to the changes of position, so that the contrast agent can fill the gastric cavity at the target area to eliminate the interference.

Strength of Recommendation: A.

\subsection{Scanning protocol [21,31,34-36]}

The basic scanning protocol includes short axial and long axial, with continuous or regional scanning.

7.3.1 From left to right: place the probe transversally in the left or left quadricostal area under the xiphoid process, and scanning is performed from cardia and gastric fundus to the middle, upper and lower stomach. Then, move the probe right to the middle and upper abdomen to scan the gastric antrum, gastric angle, middle and lower stomach. Finally, the gastric antrum, 
pylorus and duodenal bulb are scanned in the right upper abdomen. Short axial section of the gastric cavity and continuous parallel scanning are used in most cases. Because the gastric cavity in the middle stomach is relatively wide, the vertical part of the lesser and greater curvature should be scanned separately.

7.3.2 From top to bottom: place the probe laterally at the highest point of the gastric cavity, then carry out the continuous parallel scanning downward until the lowest point.

7.3.3 Continuous parallel scanning: the probe moves slowly and uniformly along the long axis or short axis of the stomach to obtain the continuous horizontal section of the stomach, which is conducive to observing the characteristics of mucosal folds and the range of lesions.

7.3.4 Cross scanning: Scanning both short axis and long axis was adopted for continuous scanning to determine the location, size and range of the lesion.

7.3.5 Fan-shaped tracking scanning: Fix one end of the probe as the fulcrum, rotate the probe as a fan-shaped to track and display the long-axis section of target area. For small lesions, it is usually difficult to target once the probe has been removed, so the fan-shaped scanning could be used to trace multiple sections of the target lesion.

Expert opinion: Lesion detection is a main purpose of gastric ultrasonography, to provide a diagnostic report including adjacent tissues and organs for clinicians, and further decide gastroscopy or other examinations. The volume of the gastric cavity increases and the gastric wall expands significantly after filling. The basic requirement for detection is to obtain continuous ultrasound images of each part of the gastric wall by adopting an appropriate scanning protocol to improve the detection efficacy.

Strength of Recommendation: A.

\section{Conventional scanning section and essentials}

\section{$[5,19,21,37,38]$}

\subsection{Section of cardia}

8.1.1 Short axis section of the cardia: (1) Scanning position: supine, standing or sitting position is preferred, and the supplementary position is right or left decubitus. (2) Scanning essentials: First, place the probe transverse and inferior to the left of the xiphoid process, ask the examinee to take a deep breath and hold. Use left lobe of the liver as the acoustic window, point the acoustic beam to the bottom of the heart. After displaying the diaphragm or the bottom of the heart, use bidirectional fan-shape scanning to display the short axis of the cardia. In this section, both the caudate lobe of the liver and the ligamentous vein fissure are important reference markers for locating the cardia, it can be detected on the left lateral side of the ligamentous vein fissure (Fig 2 ). Second, the probe was placed transverse or oblique between the 6th and 8th costal on the left side, near the midclavicular line or the front axillary line; point the acoustic beam towards the abdominal aorta for bidirectional fan-shape scanning to display the short axis section of the cardia (Fig. 3), which could display the gastric wall structure more clearly around the cardia.(3) Clinical implications: the structures of the greater, lesser curvature, anterior and posterior walls of the cardia are mainly observed. Short axial section is helpful to observe the depth of cardia cancer invasion, radial width and degree of cardia stenosis. In particular, the rate of misdiagnosis is high in tough patients with high cardia position, so careful scanning is necessary.

8.1.2 Long axis section of the cardia: (1) Scanning position: supine or standing position is preferred, and the supplementary position is right or left decubitus, which is often used for examinees with deep and high cardia position. (2) Scanning essentials: First, place the probe on the left side below the xiphoid process, ask the examinee to hold breath after deep inspiration. Use the left liver lobe as acoustic window to display the bottom of the heart, diaphragm and abdominal esophagus, fan-shape scan to detect the long axis section of the cardia (Fig. 4). Since the section of the long axis of the cardia is beak like, it is called "beak sign." Second, after displaying the long axis section of the cardia, ask the examinee to swallow or take contrast agent while conduct the scanning simultaneously to observe the smoothness of contrast agent passing through the cardia and the relaxation state of the cardia. (3) Clinical implications: mainly observe the structure of the anterior and posterior wall of the cardia, which is helpful to show the invasive cardia carcinoma, lower segment of the esophagus, achalasia and reflux of the cardia.

\section{2 section of gastric fundus}

8.2.1 Short axis section of gastric fundus: (1) Scanning position: supine position is preferred, and left decubitus is supplementary. (2) Scanning essentials: First, place the probe transversally in the left 6 th $\sim 8$ th intercostal area. After displaying the short axis section of the cardia near the midclavicular line or the front axillary line, the acoustic beam was directed towards the left diaphragmatic fornix, then use bidirectional fan-shape scanning to display the short axis section of the gastric fundus. Second, the probe was moved up one intercostal space near the axillary front line, adjust the acoustic beam directing towards the left diaphragmatic fornix to display the short axis section of the gastric fundus by using the spleen as the acoustic window (Fig. 5). Third, 
in case of imaging difficulties such as deep gastric fundus position, the acoustic frequency can be lowered and the imaging depth can be increased for scanning [32]. (3) Clinical implications: mainly observe the fundus fornix, greater curvature, anterior posterior wall of the stomach. The gastric fundus is adjacent to the left fornix of the diaphragm, which is the highest and widest area of the whole stomach, regardless of standing, sitting or supine positions. Gastric cavity gas and mucus are prone to gather in the fundus, and it is easily covered by the ribs or lung. Therefore, this is the most difficult scanning part of the whole stomach due to the acoustic interference, which requires extra attention during the examination.
8.2.2 Long axis section of gastric fundus: (1) Scanning position: supine position is preferred; left decubitus is the supplementary. (2) Scanning essentials: place the probe on the left side below xiphoid process, ask the examinee to hold breath after deep inspiration. Use the left lobe of liver as acoustic window, adjust the acoustic beam directed towards the left diaphragmatic fornix to detect left diaphragm, left lobe of liver, gastric fundus, and spleen; then move the probe to track the long axis section of the gastric fundus (Fig. 6). (3) Clinical implications: mainly to observe the fornix of the gastric fundus, anterior and posterior wall of the fundus, the middle and upper part of the stomach.
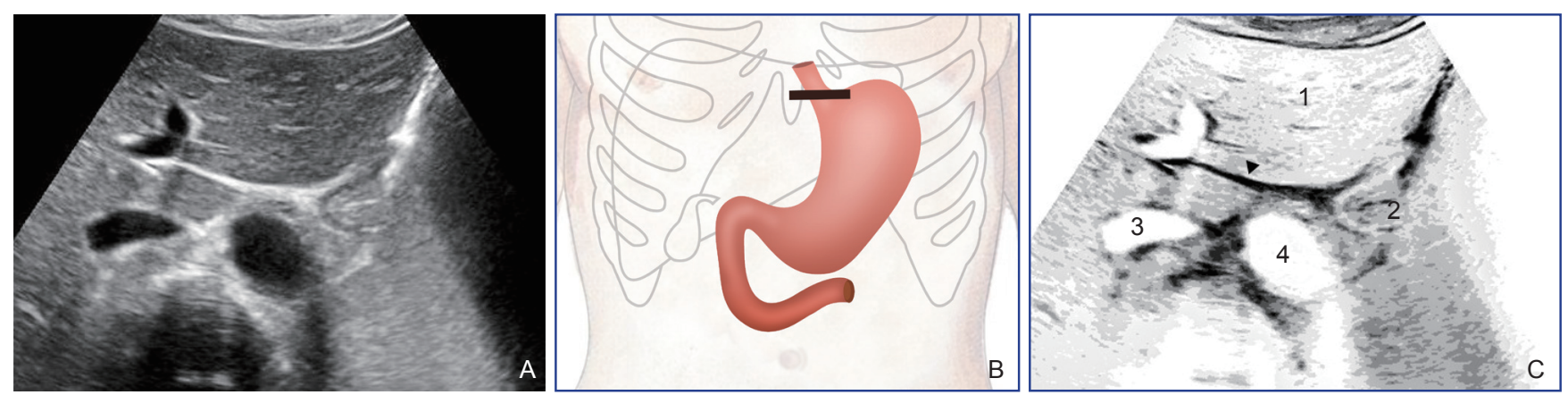

Figure 2 Ultrasonic images of short axis section of cardia and scanning schematic diagram of section (scanning under xiphoid process). A, Ultrasound image; B, Scan the schematic diagram; C, Ultrasound schematic diagram (1, Left lobe of liver; 2, Cardia; Inferior vena cava; 3, Abdominal aorta; 4, Ligamentum laceration of hepatic vein)
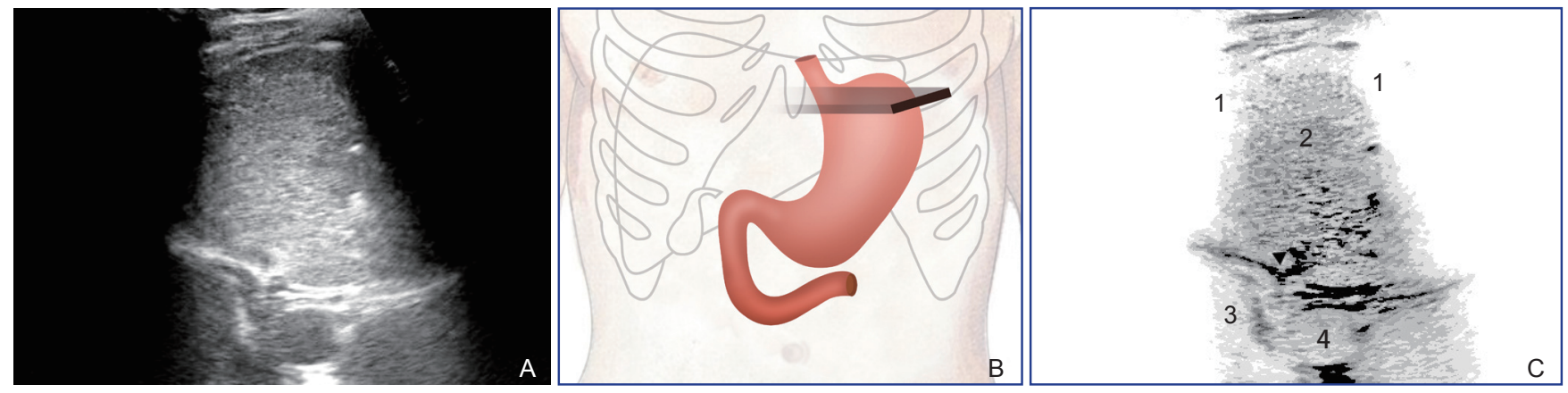

Figure 3 Schematic diagram of ultrasonic image and scanning of short axis section of cardia (scanning through left intercostal section). A, Ultrasound image; B, Scan the schematic diagram; C, Ultrasonic schematic diagram (1, Sound shadow of ribs; 2, Stomach; 3. Left lobe of liver; 4. Aorta. $\nabla$, Cardia)

\subsection{Section of the gastric body}

8.3.1 Short axial section of the middle and upper part of the gastric body: (1) Scanning position: standing position is preferred, and supine position is supplementary. (2) Scanning essentials: First, place the probe transversely or obliquely on the left side of the 6th $\sim 8$ th intercostal, midclavicular line or anterior axillary line for scanning. The acoustic beam is perpendicular to the abdomen. After displaying the short axis section of the cardia, move the probe downward for continuous parallel scanning to display the short axis section of the middle and upper part of the stomach. Second, place the probe transversely under the left side of xiphoid process to display the short axis section of the cardia, continue the scanning downward to display the short axial section of the middle and upper gastric body (Fig. 7). Most of the short axis sections of the middle and upper gastric body presented a quasi-circular structure. (3) Clinical implications: Mainly to observe the structure of the middle and upper stomach, including the greater and lesser curvature, anterior and 
posterior walls. However, it should be noted that in this section, the angle between the gastric wall structure and the acoustic beam in the greater and lesser curvatures is very small or nearly parallel, the echoic loss may result in the unclear display of the gastric wall layers in the greater and lesser curvatures, which predispose misdiagnoses to be made.
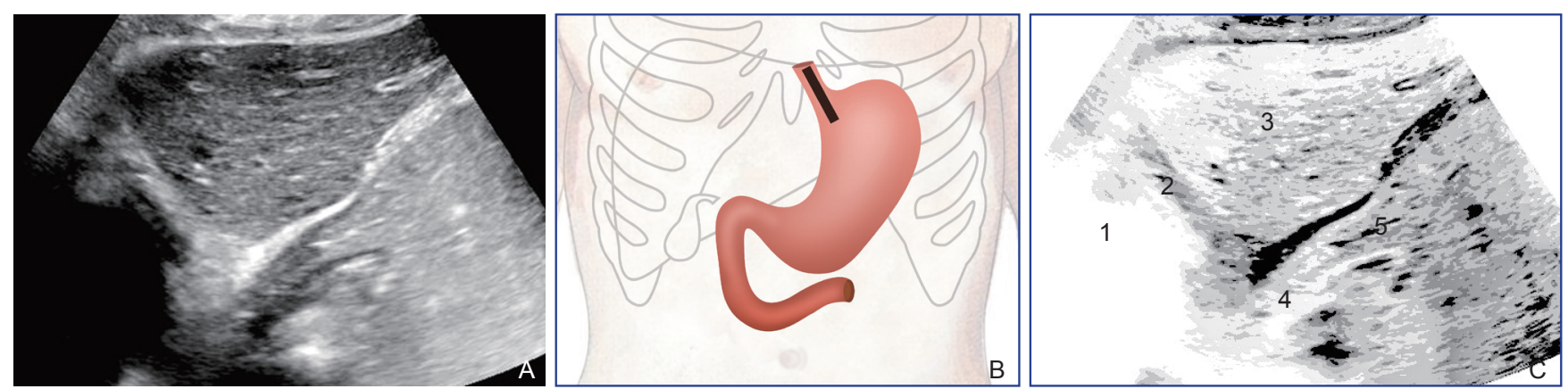

Figure 4 Ultrasonic images and scanning schematic diagram of section of long axis of cardia (scanning under xiphoid process). A, Ultrasound image; B, Scan the schematic diagram; C, Ultrasound Schematic Diagram (1, 2, Diaphragm; 3, Left lobe of liver; 4, Abdominal esophagus; 5, Cardia)
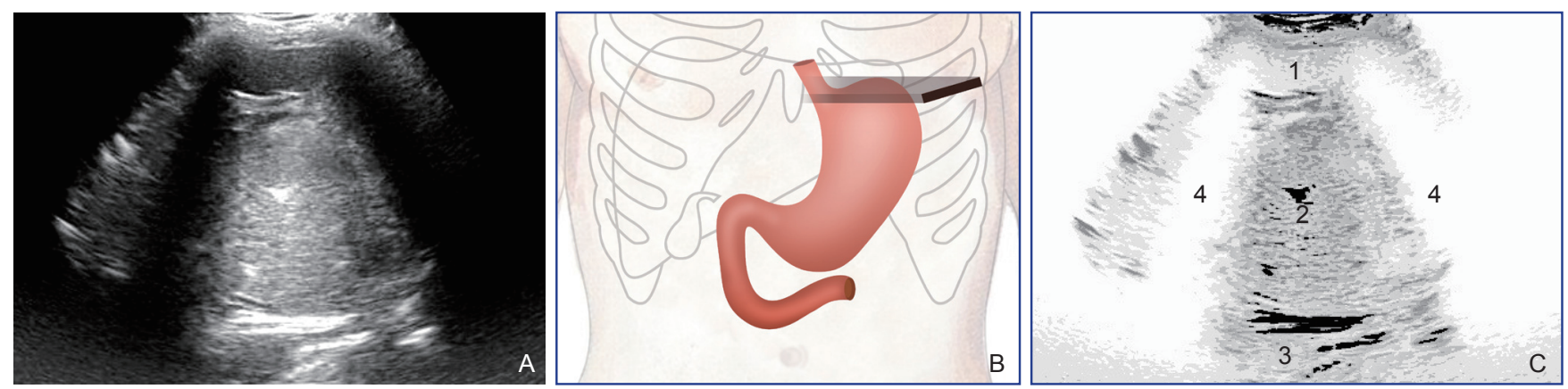

Figure 5 Short-axis section of the gastric fundus ultrasonic image and schematic diagram of the canning (scanning through the left intercostal section). A, Ultrasound image; B, Schematic scanning diagram; C, Ultrasound schematic diagram (1, Spleen; 2, Gastric fundus; 3, Aorta; 4, Acoustic shadow of ribs)
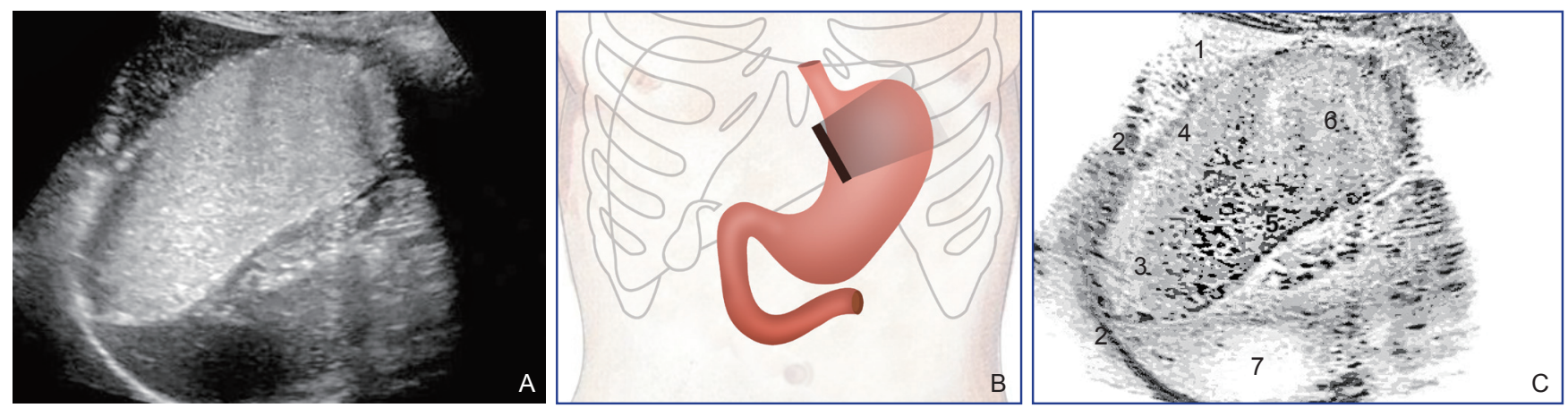

Figure 6 Ultrasonic images of long axial section of the gastric fundus and schematic diagram of the section (scanning under xiphoid process). A, Ultrasonic image; B, Scan the schematic diagram; C, Ultrasound schematic diagram (1, Left lobe of liver; 2, Left diaphragm; 3, The gastric fundus; 4, Anterior wall of the stomach; 5, Posterior wall of the stomach; 6, Stomach; 7, Spleen)

8.3.2 Short axis section of the middle and lower gastric body: (1) Scanning position: standing position is preferred; supine position is the supplementary. (2) Scanning essentials: After displaying the short-axis section of the middle and upper gastric body, continue the parallel scanning downward to display the short-axis section of the middle and lower part (Fig. 8). The shortaxis section of the middle and lower gastric body mostly presents an elliptical structure. In the cases of high gastric cavity volume, it is difficult to display the greater curvature and lesser curvature simultaneously in one section, so it is necessary to move the probe laterally for complete scanning. (3) Clinical implications: Mainly to observe the structure of the middle and lower part of the stomach, including the greater and lesser curvatures, the anterior and posterior walls. 

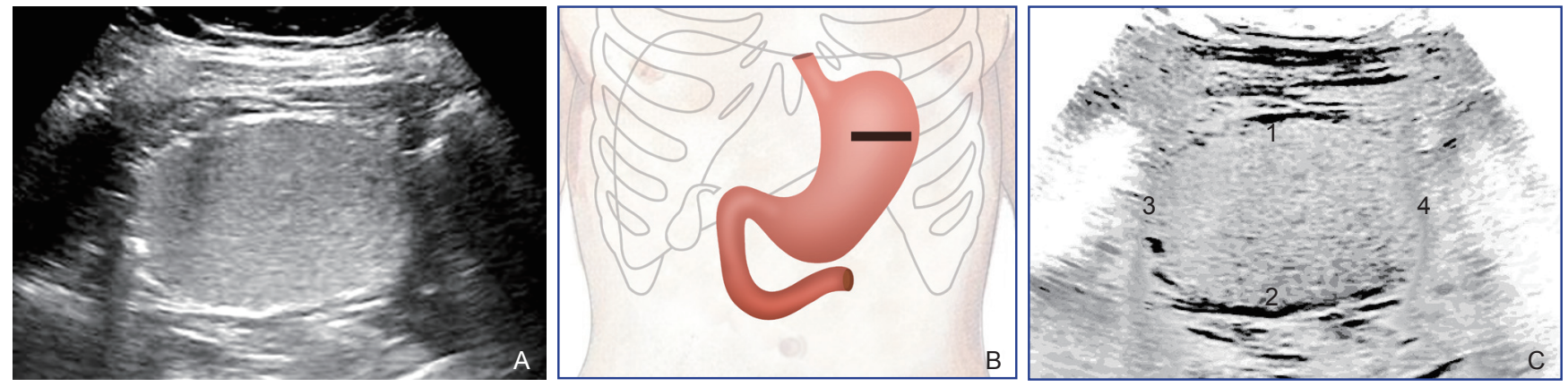

Figure 7 Short-axis section of the middle and upper part of the gastric body ultrasound images and scan schematic diagram of the section (scan under xiphoid process on the left side). A, Ultrasound image; B, Scan the schematic diagram; C, Schematic diagram of ultrasound (1, Anterior wall of the stomach; 2, The posterior wall of the gastric body; 3, Lesser curvature of the gastric body; 4, Greater curvature of the gastric body)
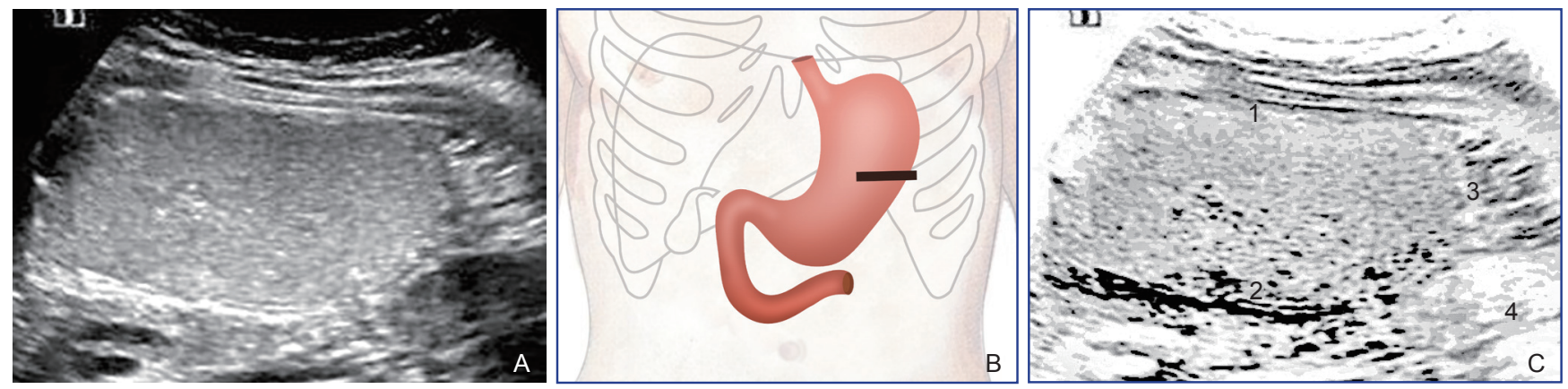

Figure 8 Short-axis section of the middle and lower gastric body and schematic diagram of the section (scan under the left side of xiphoid process). A, Ultrasound image; B, Schematic diagram of scanning; C, Schematic diagram of ultrasonogram (1, Anterior wall of the stomach; 2 , The posterior wall of the stomach; 3, Greater curvature; 4, Left kidney)

\subsection{Sections of gastric angle}

8.4.1 Long axis section of gastric Angle: (1) Scanning position: standing position is preferred; supine position or right-side decubitus is supplementary. (2) Scanning essentials: place the probe transversally under the xiphoid process, adjust acoustic beam perpendicularly to the abdomen. Continuous parallel scan to show the horizontal " 8 " shape structure composed of the gastric antrum and the gastric body, which is the long axis section of the gastric angle (Fig. 9). (3) Clinical implications: mainly to observe the gastric antrum, anterior and posterior wall structure of the lesser curvature. The confluence area of gastric antrum and gastric body is the gastric Angle, which marks the anatomical boundary between gastric antrum and gastric body. Gastric angle is the most common site of ulcer and gastric cancer, which call for extra attention.
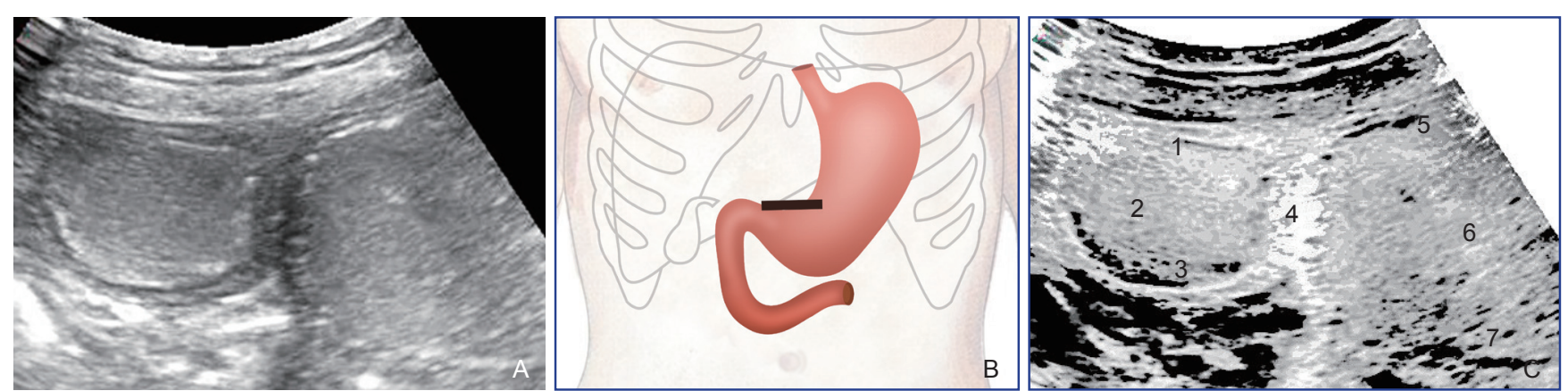

Figure 9 Ultrasonogram and schematic diagram of the long axial section of the gastric angle (scanning under xiphoid process, Angle notch horizontal). A, Ultrasonogram; B, Schematic diagram; C, Schematic diagram of ultrasound (1, Anterior wall of gastric antrum; 2, Gastric antrum; 3, Posterior wall of gastric antrum; 4, The stomach angle; 5, Anterior wall of the stomach; 6, The gastric body; 7, The posterior wall of the stomach)

8.4.2 Short axis section of gastric angle: (1) The scanning position: standing position is preferred; supine or right-side decubitus is supplementary. (2) Scanning essentials: rotate the probe clockwise to make the probe 
perpendicular to the long axis of the gastric corner to display the short axis section of the gastric angel (Fig. 10). (3) Clinical implications: mainly to observe the gastric angle, including the corresponding area such as the lesser curvature, greater curvature, anterior and posterior walls.
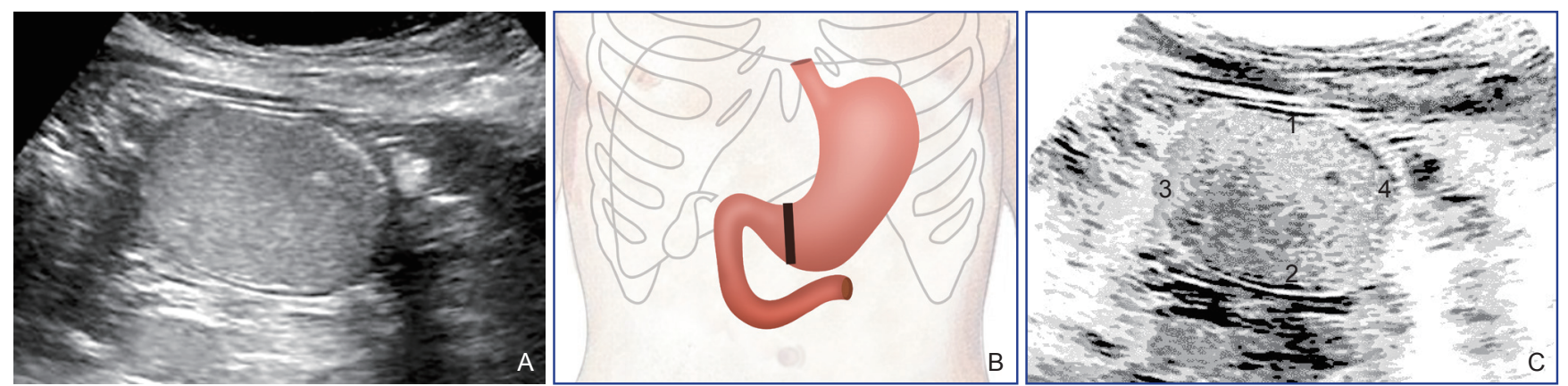

Figure 10 Ultrasonogram and schematic diagram of the short axial section of the gastric angle (scanning under xiphoid process). A, Ultrasonogram; B, Schematic diagram; C, Schematic diagram of ultrasound (1, Anterior gastric wall;2, Posterior gastric wall;3, Lesser curvature; 4, Greater curvature)

8.4.3 Coronal section of gastric angle: (1) Scanning position: standing position is preferred, right side decubitus or supine position are supplementary.(2) Scanning essentials: First, remember the probe position when displaying the horizontal " 8 " shape structure of the gastric angle, then move the probe downward to the greater curvature (as the upper left or lower left umbilicus), point the acoustic beam at the gastric angle from bottom to top to scan the "8" shape structure horizontally through a front and back fan-shape (similar to the scanning of the oblique section of the right liver under the right costal margin), to display the coronal section of the gastric angle (Fig. 11). Second, move the probe back and forth along the greater curvature to perform a multi-point coronal section of the gastric angle. (3) Clinical implications: Mainly to observe the wall structure of gastric angle, the greater and lesser curvatures. Gastric angle can be divided into three sides, including gastric antrum, angle and stomach side. The main advantage of coronal scanning is that the acoustic beam projected vertically, which is the key to improve the imaging clarity of gastric wall hierarchy. The conspicuity of the acoustic angle is related to the position and the shape of the stomach. For example, it is conspicuous in cases of hook-shaped and ptotic stomach, or in someone standing, while obscure in ox horn stomach.
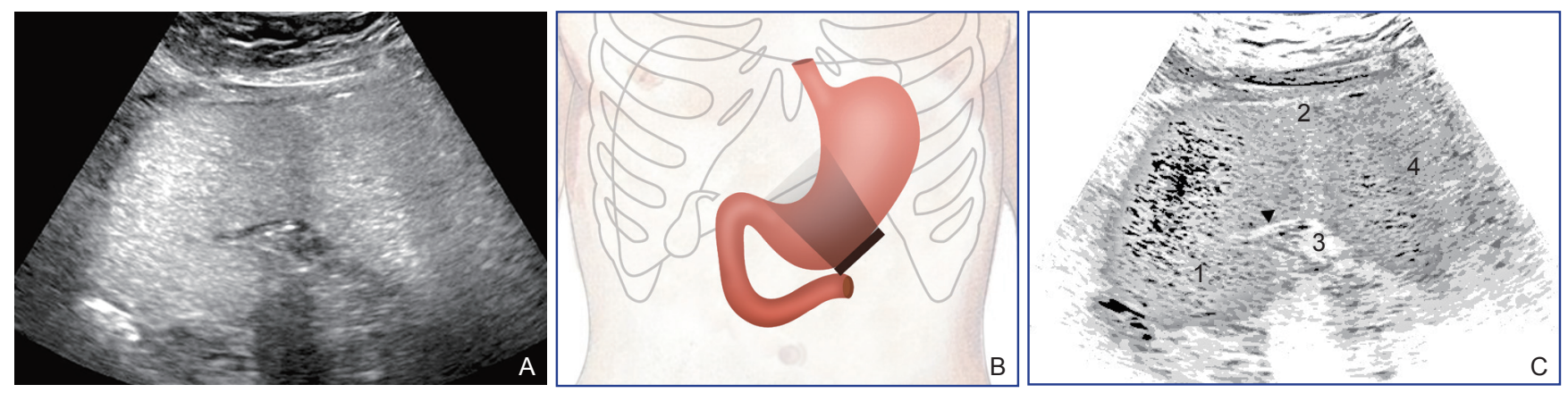

Figure 11 Ultrasonogram and schematic diagram of coronal section of the gastric corner (scan through the upper left umbilicus). A. Ultrasonogram. B. Schematic diagram, C. Schematic diagram of ultrasound (1. Anterior wall of stomach, 2. Great curvature, 3. Lesser curvature, 4. Gastric body; $\boldsymbol{\nabla}$ stomach angle)

\subsection{Section of gastric antrum}

8.5.1 Long axis section of gastric antrum and gastric body: (1) Scanning position: standing position is the preferred, supine or right-side decubitus is supplementary. (2) Scanning essentials: after displaying the long axis section of the gastric angle, move the probe downward for continuous parallel scanning to display the maximum long axis section of the gastric antrum and gastric body (Fig. 12). (3) Clinical implications: mainly to observe the structure of the gastric body and the antrum.

8.5.2 Long axis section of the gastro-duodenal bulb: (1) Scanning position: standing position is preferred; supine or right decubitus are supplementary. (2) Scanning essentials: First, place the probe horizontally in the right upper abdomen to show the gastric antrum around 
the pancreatic head, rotate the probe clockwise and slantingly in the right upper abdomen, to track the long axis section of the gastric antrum and duodenal bulb (Fig. 13). Then place the probe slantingly in the right upper abdomen, using the right liver lobe and the gallbladder as acoustic window to display the long axis section of the gastro-duodenal bulb. After displaying the long axis section of the gastric angle, move the probe upward to display the structure of the gastric antrum, then track the long axis section of the gastric antrum to the duodenal bulb. (3) Clinical implications: mainly to observe the anterior and posterior walls of gastric antrum, pylorus, duodenal bulb, pyloric lateral structure of the bulb, to investigate the passage of pyloric contrast agent and gastric mucosa prolapse, and whether the contents of the duodenal bulb, including the mucosa of the bulb, reflux through the pylorus to the gastric antrum. Gastric antrum is a common site for inflammation, ulcers and tumors, which should be examined carefully.
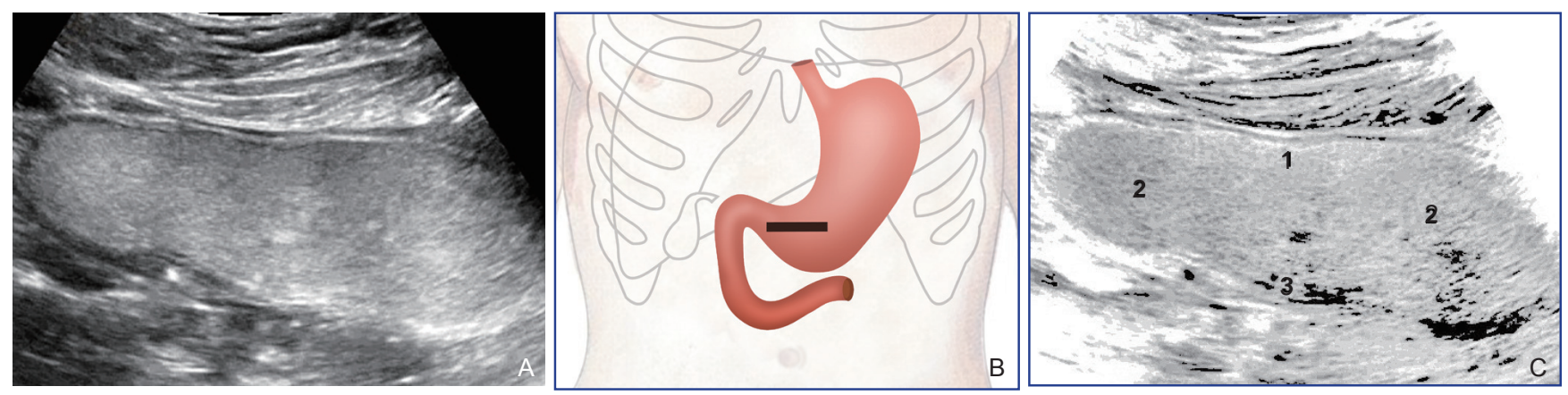

Figure 12 Ultrasonogram and schematic diagram of long axis section of gastric antrum and gastric body (scan of the middle and upper abdomen). A. Ultrasonogram. B. Schematic diagram, C. Schematic diagram of ultrasound (1. Anterior gastric wall; 2. Gastric cavity; 3. Posterior gastric wall)
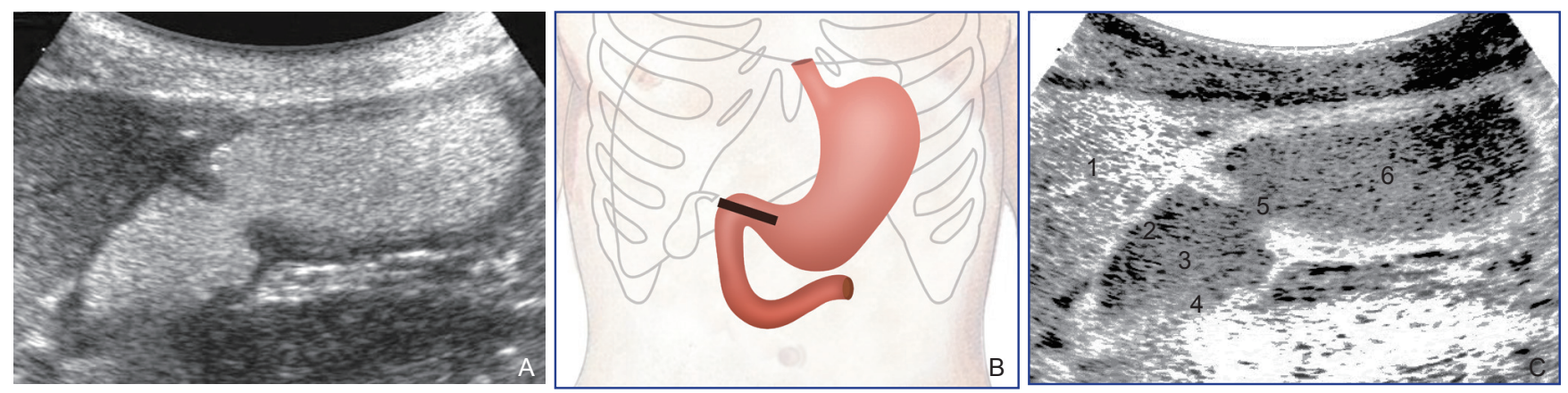

Figure 13 Ultrasonogram and schematic diagram of long axis of gastro-duodenal bulb and ultrasonic image (scan through right upper abdomen). A, Ultrasonogram; B, Schematic diagram; C, Schematic diagram of ultrasound (1, Right liver lobe; 2, Anterior wall of bulb; 3, Duodenal bulb; 4, Posterior wall of bulb; 5, Pylorus; 6, Gastric antrum)

8.5.3 Short axis section of gastric antrum: (1) Scanning position: standing position is preferred; supine position or right-side decubitus is supplementary. (2) Scanning essentials: First, display the long axis section of the gastro-duodenal bulb, then rotate the probe clockwise to be perpendicular to the long axis of the gastro-duodenal bulb to display the short axis section of the gastro-antrum (Fig. 14). Second, perform a continuous short axial scanning of the entire gastric antrum (from the stomach angle to pylorus). (3) Clinical implications: Mainly to observe the structures of lesser curvature, greater curvature, anterior wall and posterior wall of the gastric antrum. Because the tubular structure of the gastric antrum is relatively long, and the long axis section cannot show two curvatures entirely, it is important to combine with the short axis section during the scanning, so as not to miss gastric antrum lesions. However, attention should also be paid to the echoic loss artifact of the lesser and greater curvatures of the gastric wall.

8.5.4 Coronal section of the gastro-duodenal bulb: (1) Scanning position: standing position is the preferred, supine or right decubitus are supplementary positions. (2) Scanning essentials: remember the position of the probe when displaying the section of the long axis of the gastro-duodenal bulb, then move downward near the descendent duodenum, point the acoustic beam in the direction of the gastric antrum and the bulb, perform a bidirectional fan-shaped scanning to display the coronal 
section of the gastro-duodenal bulb (Fig. 15). (3) Clinical implications: gastro-duodenal bulb coronary section is important to display pylorus, which is difficult to acquire by gastroscope. In addition, the coronal section of the gastro-duodenal bulb could show the relationship to adjacent structures including the gallbladder, duodenum and gastric antrum, which is useful for the differential diagnosis of endoscopic "submucosal lesions" caused by gallbladder enlargement displacing the duodenal bulb and the gastric antrum.
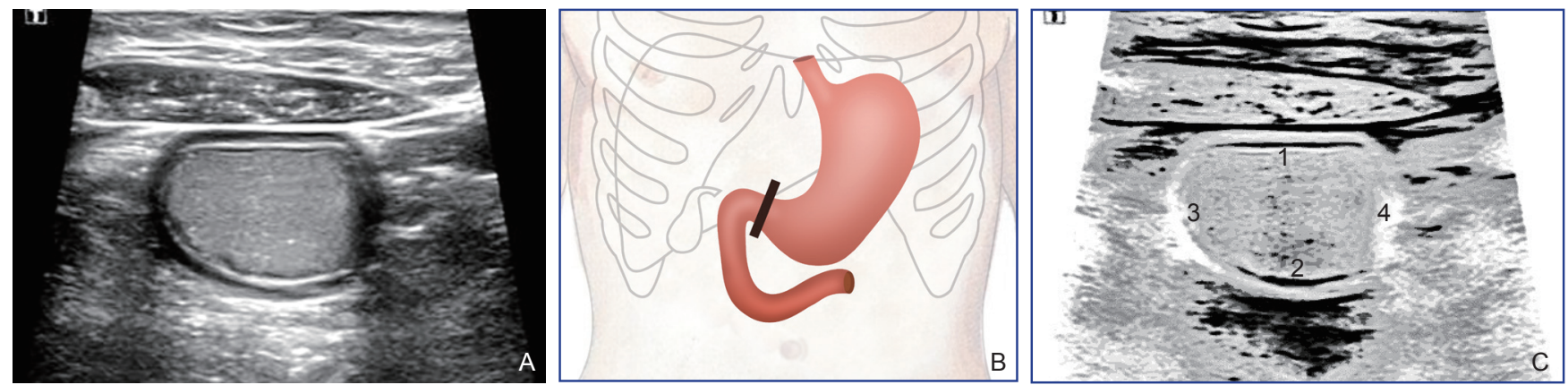

Figure 14 Ultrasonogram and schematic diagram of short-axis section of gastric antrum (scan through right upper abdomen). A, Ultrasonogram; B, Schematic diagram; C, Schematic diagram of ultrasound (1, Anterior wall of gastric antrum; 2, Posterior wall of gastric antrum; 3, Lesser curvature of gastric antrum; 4, Greater curvature of gastric antrum)
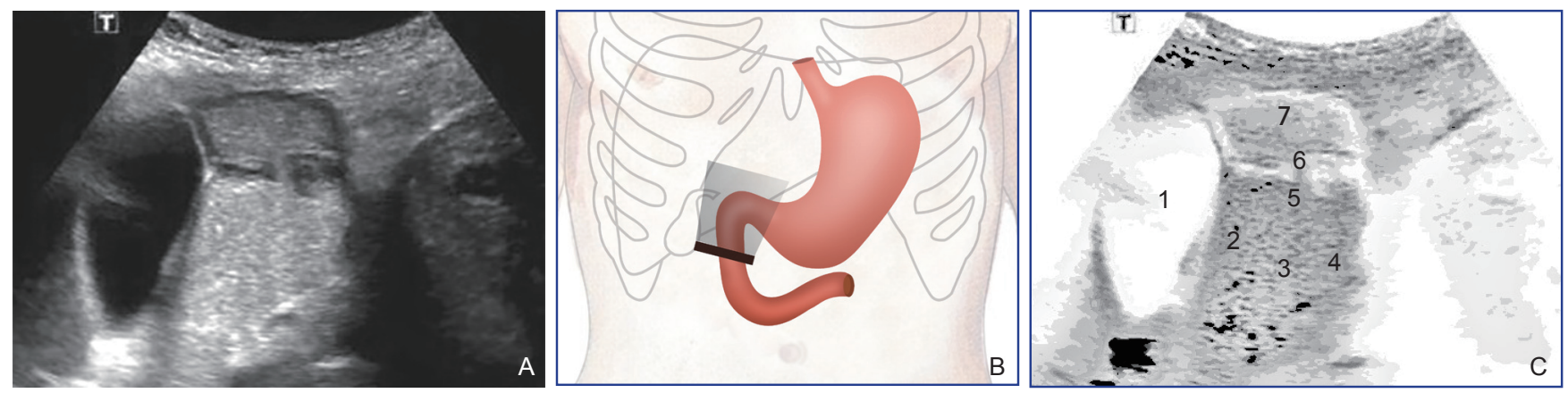

Figure 15 Ultrasonogram and schematic diagram of coronal section of the gastro-duodenal bulb (scan through the right upper abdomen)。 A, Ultrasonogram; B, Schematic diagram; C, Schematic diagram of ultrasound (1, Gallbladder; 2, Lesser curvature side of the bulb; 3, Duodenal bulb; 4, Greater curvature side of the bulb; 5, Pyloric side of the bulb; 6, Pylorus; 7, Gastric antrum)

\subsection{Long axial section of descendent duodenum}

8.6.1 Scanning position: Standing position is preferred; supine and right decubitus are the supplementary positions.
8.6.2 Scanning essentials: After displaying the long axis section of the gastro-duodenal bulb, slant the probe to display the long axis section of the descendent duodenal bulb (Fig. 16).
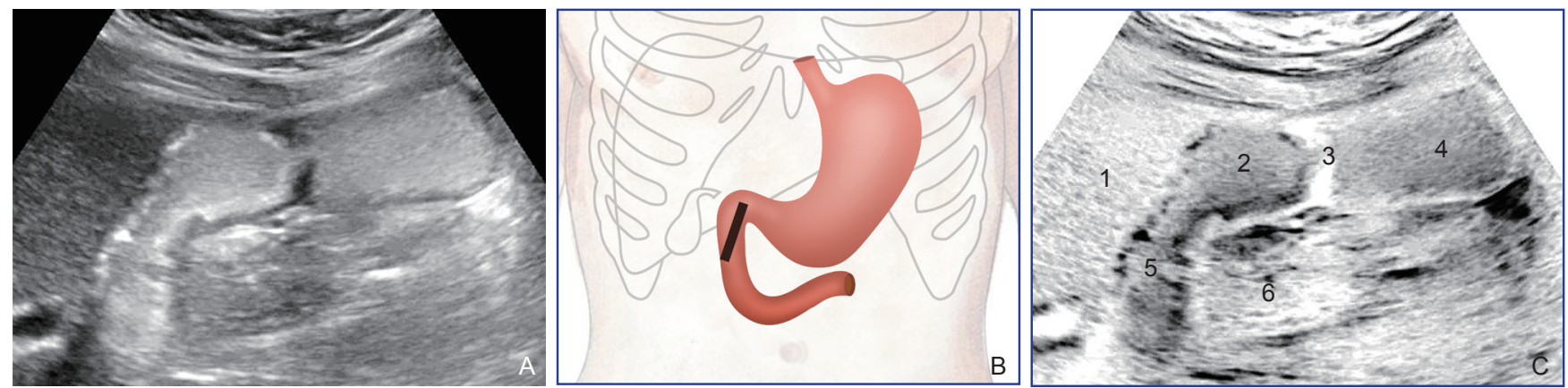

Figure 16 Ultrasonogram and schematic diagram of the long axis section of descendent duodenum (scan through the right upper abdomen)。 A, Ultrasound image; B, Scan the schematic diagram; C, Ultrasound schematic diagram (1, Right liver lobe; 2, Duodenal bulb; 3, Pylorus; 4, Gastric antrum; 5, Descendent duodenum; 6, Head of pancreas) 
8.6.3 Clinical implications: mainly to observe the intestinal wall structure of the descendent duodenum and the filling characteristics of the intestinal lumen.

\subsection{Horizontal long axis section of duodenum}

8.7.1 Scanning position: supine position is preferred, standing and sitting are supplementary positions.

8.7.2 Scanning essential points: After display the long axis section of gastric antrum and stomach body, continue the parallel scanning towards the umbilicus to acquire the horizontal long axis section of duodenum between the superior mesenteric artery and aorta (Fig. 17).
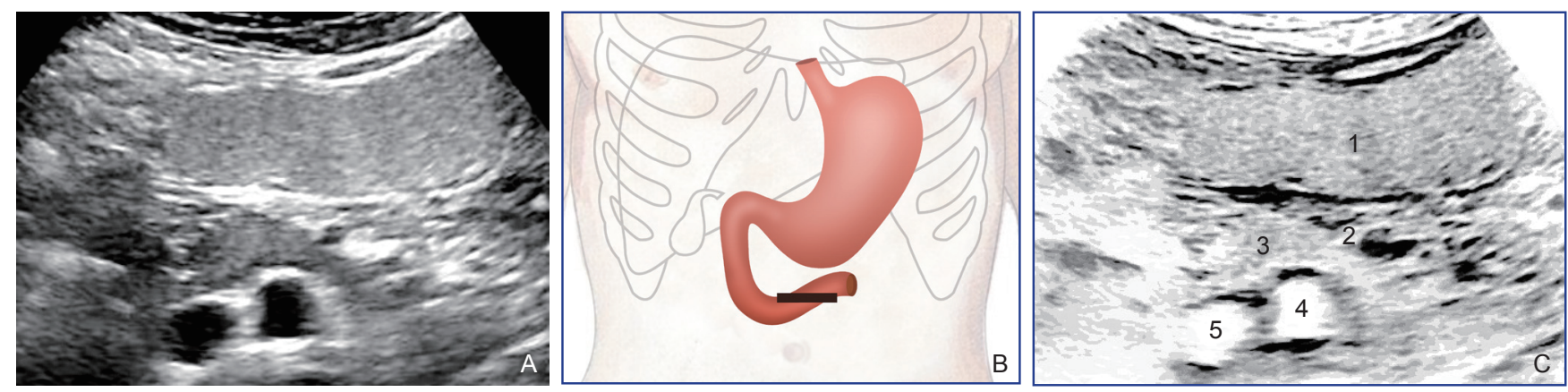

Figure 17 Ultrasonic image and scan schematic diagram of the horizontal long axis section of the duodenum (scan of the middle and upper abdomen). A, Ultrasound image; B, Scan the schematic diagram; C, Schematic diagram of ultrasound (1. Gastric body; Superior mesenteric artery; 2. 3. Duodenal level; Abdominal aorta; 4. 5. The inferior vena cava)

8.7.3 Clinical implications: Mainly to observe the intestinal wall structure and the filling characteristics of intestinal lumen at the level of duodenum.

Expert opinion: The above items summarize the common sections of gastric ultrasonography, scanning essential points and the characteristics of the sonograms, which guides to establish the gastric ultrasonographic standardization, which needed verified in further clinical application. In addition, the examiner should clearly aware that stomach is the largest lumen structure of the digestive tract, the overall morphological and structural characteristics of the stomach is hard to display by any single section during the scanning. It is important to obtain a series of ultrasonograms to establish a comprehensive three-dimensional perspective of gastric ultrasonography.

Strength of Recommendation: A.

\section{Quality control}

\subsection{Empty stomach cavity}

Since food residues and mucus in the stomach can interfere the display of the gastric wall, gastric cavity and lesions, examinee should be fasting and refrain from drinking before examination.

\subsection{Flow filling}

With changes of positions, the contrast agent flows in the gastric cavity and fills the target area. The flow filling is also an important way to find the best position for displaying lesions [39].

\subsection{Display in sections}

Usually, the gastric cavity, or even a part of the stomach, cannot be completely shown on a single section of the sonogram in the same position. Therefore, it is necessary to scan and display each part of the stomach in a certain order without omission.

\subsection{Enlarged display}

The normal thickness of the gastric wall is about $3 \sim 5 \mathrm{~mm}$, and each layer of the gastric wall is even thinner. Under the premise of imaging quality, the gastric sonograms should be fully enlarged for observation or measurement [40]. High frequency probe can also be used to observe details accordingly.

\subsection{Moderate pressure application [21,28,41]}

In order to reduce the interference of gastrointestinal gas, appropriate probe pressure upon the scanning area could reduce the imaging distance of gastric cavity or gastric wall, provides better focus and visual field.

\subsection{Breathing control}

To improve the quality of gastric ultrasound imaging, breathing control methods such as breath-holding after deep inhalation are used to displace the stomach downwards, as well as push aside the surrounding intestines.

\subsection{Examination time}

Adequate examination time can improve the detection rate of lesions [42]. Usually, it takes $10 \sim 15 \mathrm{~min}$ per examinee to accomplish a high-quality gastric ultrasound scanning.

\subsection{All sections complement each other}

9.8.1 All parts of the stomach are of "pipeline" 
structures, and the long axis section shows "parallel pipeline" sign, which is used to observe the anterior and posterior gastric wall structure. The short axial section shows "target ring" sign, which is used to observe the greater curvature, the lesser curvature, and the structure of the anterior and posterior walls [41].

9.8.2 The long axis or short axis section of the stomach is not simply scanned by placing the probe longitudinally or transversely in the abdomen, but by adjusting the direction of the probe or the acoustic beam angle. The long axis or short axis section is consistent with the direction of the long axis or short axis of the stomach anatomy.

9.8.3 It is necessary to evaluate the location, range and depth of gastric lesions through all relevant sections to make final, comprehensive diagnosis.

\subsection{Gastroscopy and ultrasound follow-up}

9.9.1 Gastric ultrasonography can display lesions, but generally cannot clearly diagnose benign or malignant lesions. Therefore, after the lesion is detected by ultrasound, endoscopic gastric mucosal biopsy should be arranged as soon as possible for pathological diagnosis.

9.9.2 For cases with obesity or high gastric position, corresponding descriptions should be mentioned in the report, and gastroscopy or other imaging examinations should not be delayed for diagnosis.

9.9.3 Examiners should follow-up and integrate the subsequent results of gastroscopy, endoscopic ultrasound, $\mathrm{CT}$, pathology and other related information, to further improve the quality of gastric ultrasound diagnosis constantly.

9.9.4 For people at risk of gastric cancer, the ultrasonic follow-up period should be appropriately shortened, such as no less than once a year to facilitate early detection and diagnosis of malignancy.

Expert opinion: The above items summarized the key points of quality control of gastric ultrasonography, which is important for carrying out high-quality examination and ensuring medical safety.

Strength of Recommendation: B.

\section{Measurement Method and Normal Reference}

\section{Value}

\subsection{Measurement method}

10.1.1 Measurement of normal stomach: (1) Cardia: the inner diameter of the cardia and the thickness of the tube wall are measured by freezing the image when the contrast agent passing through. (2) Gastric wall: the thickness of the gastric wall is commonly measured when gastric cavity is moderately filled. Adjust the acoustic beam perpendicular to the gastric wall then measure the vertical diameter between the mucosal surface and serosal layer. In the long axis section of the stomach, parallel and adjacent gastric mucosal folds may lead to overestimate of gastric wall thickness [28]. Therefore, suspected thickening of gastric wall should be measured and confirmed in the short axial section. (3) Pylorus: measure the inner diameter when the pylorus is relaxed. 10.1.2 Localization of gastric lesions :(1) Localization of lesions according to anatomy, such as the cardia, gastric horn and gastric antrum. (2) Use the "cross" scanning method to locate the lesion. (3) In the short axis section of the stomach, keep the greater curvature side at $3 \mathrm{o}$ 'clock, the lesser curvature side at $9 \mathrm{o}$ 'clock, the anterior wall at $12 \mathrm{o}$ 'clock, and the posterior wall at $6 \mathrm{o}$ 'clock, then describe the lesions according to the "clock position"[43].

10.1.3 Measurement of gastric lesions: (1) Length: the maximum length of gastric wall lesions is measured on the long axis section (Fig. 18). (2) Height: the maximum height gastric wall lesions are measured on the short axis section. Small lesions can be measured using linear measurement functional detection. Segmental linear measurement function detection (Fig. 19) or TRACE measurement function detection can be used for greater lesions. Large lesions can be described by circumferential semi-quantitative method. (3) Thickness: the thickness of gastric wall lesions is measured on the long axis or short axis section. (4) Circumferential diameter: For patients with large gastric wall lesions, the scope of gastric wall lesions could be evaluated on the short-axis section, such as 1/3,1/4 circumference. (5) Others: measuring the maximum diameter line of gastric wall depression or eminence lesions, etc.

\section{Recommendation strength: $B$.}

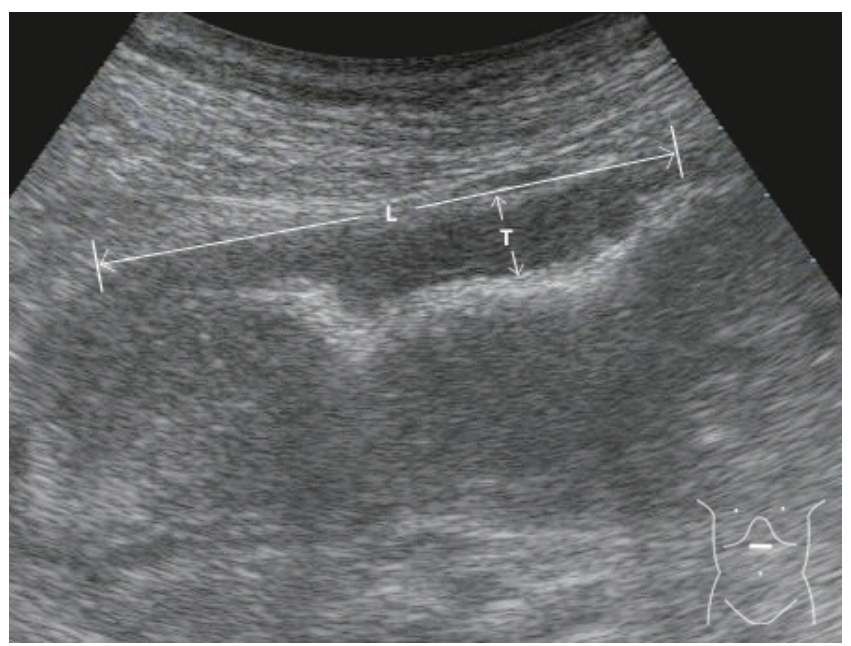

Figure 18 Length and thickness measurement of gastric wall lesions L, Length; T, Thickness 


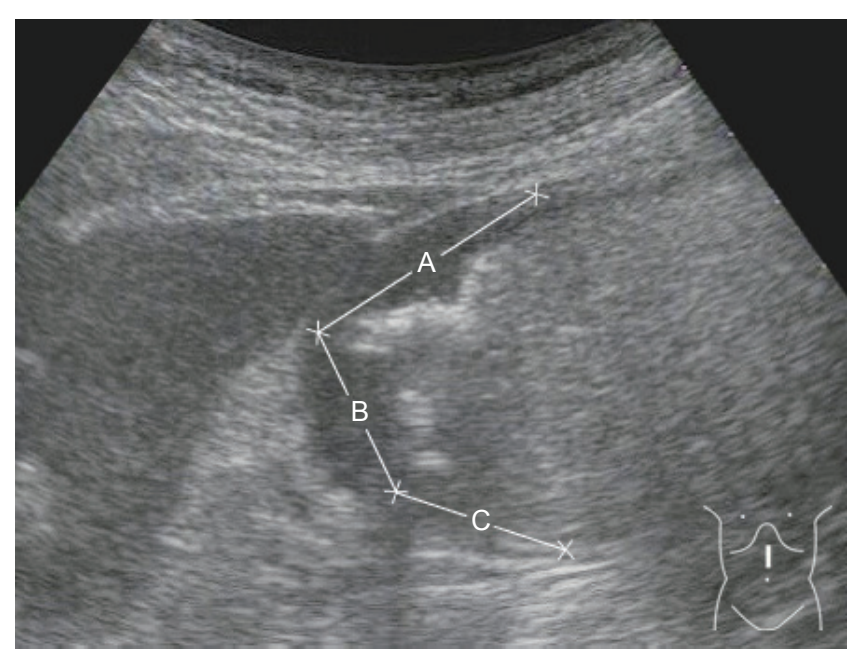

Figure 19 Segmented measurement of upper and lower diameter of gastric wall lesion (upper and lower diameter of lesion $=\mathrm{A}+\mathrm{B}+\mathrm{C}$ )

\subsection{Normal reference of measurement [7, 18, 20, 23,} 44-46]

The normal reference of gastric measurement is still controversial. The data of normal reference values for gastric measurement in this consensus are mainly cited from literatures published in China.

10.2.1 Cardia: inner diameter $\leq 1.5 \mathrm{~cm}$, the thickness of the tube wall $0.3 \sim 0.5 \mathrm{~cm}$.

10.2.2 Gastric wall: normal gastric wall thickness is gastric antrum $>$ gastric body $>$ gastric fundus, gastric antrum wall thickness $0.4 \sim 0.6 \mathrm{~cm}$ (neonatal $<0.4 \mathrm{~cm}$ ), wall thickness of the gastric body is $0.3 \sim 0.5 \mathrm{~cm}$, thickness of gastric wall in fundus $\leq 0.3 \mathrm{~cm}$.

10.2.3 Pylorus: adult pylorus inner diameter 0.2 $0.6 \mathrm{~cm}$, length $0.5 \sim 0.8 \mathrm{~cm}$. In infants, the length of pyloric canal $<1.5 \mathrm{~cm}$, the thickness of pylorus $<1.1 \mathrm{~cm}$, and thickness of pyloric muscle layer $<0.25 \mathrm{~cm}$.

10.2.4 Lower gastric boundary: When standing, the lowest point is generally located above the umbilicus.

10.2.5 Thickness of duodenum wall: $0.3 \sim 0.5 \mathrm{~cm}$.

\section{Strength of Recommendation: C.}

\section{Image Recording and Storage}

\subsection{Standardization [47]}

The value of standardized image recording and storage is multifarious, including the preservation of important medical information for legal disputes, training and teaching materials, and retention and quality controls.

\subsection{Image storage amount and detection rate of gastric diseases}

Image storage amount is correlated with the detection rate of gastric diseases [48]. Storage of images may slow down the scanning speed, so a balance between the number of images stored and the inspection speed should be considered.

\section{3 $" 6+X "$ storage mode}

It is recommended that no less than 6 images be kept for each subject, including : (1) long axis or short axis section of the cardia; (2) long axis section of the gastric fundus; (3) short axis section of the middle and lower part of the gastric body; (4) long axis section of the gastric angle; (5) coronal section of the gastric angel; (6) long axis section of the gastro-duodenal bulb; "X" refers to the amount of images stored at the lesion site, and at least 2 or more "cross-sectional" images of the lesion are stored. In addition, attention should be paid to the preservation of cine for retrospection and analysis, especially those of the cardia, gastric angle and antrum.

Strength of Recommendation: B.

\section{Qualification and Training [47]}

\subsection{Qualification access}

12.1.1 The examiner shall have certain clinical experience and obtained the People's Republic of China Certificate of Physician Qualification and Certificate of Practicing Physician. The scope of clinical practice shall be medical imaging and radiotherapy.

12.1.2 Operation ability is the core of gastric ultrasonography. Examiners should master the basic theory, knowledge and skills of ultrasound examination.

\subsection{Trainings}

12.2.1 To carry out this examination, examiner must attend the training course and pass the board of gastrointestinal and/or abdominal ultrasound diagnosis approved by the national or provincial municipal authorities.

12.2.2 The examiner should master the knowledge of gastric anatomy and pathophysiology, and be familiar with the basis of clinical diagnosis and treatment of gastric diseases.

12.2.3 The examiner should receive the clinical courses of gastric ultrasonography, and have practiced the examination for more than 50 cases.

Expert opinion: Gastric ultrasonography is an operation-dependent diagnostic technique. In order to reduce misdiagnoses, the admission and training system should be carefully implemented to carry out this examination.

Strength of Recommendation: B.

\section{Funding}

The article was funded by Shanghai Medical Leading Talents Training 
Program (2019LJ21), Shanghai Key Clinical Specialty Construction Project of "Focus Subject" (SHSLCZDZK03502) and Shanghai Engineering Research Center of Ultrasound Diagnosis and Treatment (19DZ2251100)

\section{Declaration}

The contents of this article in Chinese were peerreviewed and accepted by the Chinese Journal of Medical Ultrasound (Electronic Edition) for 2020 17(10):933952. This English version of the consensus was adapted from the Chinese one with permission. The link of Chinese vision of the article as follows: https://kns.cnki. net $/ \mathrm{kcms} /$ detail/detail.aspx?FileName=ZHCD202010003 $\&$ DbName $=$ CJFQ2020

\section{Conflict of Interests}

There is no conflict of interests to declare for between all authors and relevant academic committees.

\section{Expert review}

Jianquan Zhang, Weiwei Zhan, Hong Ding

\section{Writing Experts}

Li Shen, Jianquan Zhang, Xingang Gu, Chang Liu

\section{Preparation of Expert Group Members (alphabetical order by surname)}

Hongyan Chen, $M D^{a}$, Hong Ding, $M D^{b}$, Lianfang $D u, M D^{c}$, Chao Fang, $M D^{d}$, Xingang $G u, M D^{e}$, Liqiong Jia, $M D^{f}$, Lixin Jiang, $M D^{g}$, Yuming Jin, $M D^{h}$, Chang Liu, MD ${ }^{i}$, Feng Pei, $M D^{j}$, Li Shen, $M D^{k}$, Jingfang Shi, $M D^{l}$, Liping Sun, $M D^{m}$, Yingjie Sun, $M D^{n}$, Yingchun Wang, $M D^{\circ}$, Huixiong $X u, M D^{p}$, Mei $Y u, M D^{q}$, Weiwei Zhan, $M D^{r}$, Jianquan Zhang, $M D^{s}$, Li Zheng, $M D^{t}$

${ }^{a}$ Minhang Hospital Affiliated to Fudan University (Minhang Central Hospital of Shanghai); ${ }^{b}$ Huashan Hospital Affiliated to Fudan University; ${ }^{c}$ First People's Hospital Affiliated to Shanghai Jiao Tong University, School of Medicine; ${ }^{d}$ Shanghai Xuhui District Central Hospital; ${ }^{e}$ Putuo Hospital Affiliated to Shanghai University of Traditional Chinese Medicine; ${ }^{f}$ Wusong Hospital, Zhongshan Hospital, Fudan Medical University; ${ }^{g}$ The Renji Hospital Affiliated to Shanghai Jiao Tong University; ${ }^{h}$ Kangjiang Hospital, Yangpu District, Shanghai, ${ }^{i}$ Shanghai Tenth People's Hospital, Ultrasound Research and Education Institute, School of Meicine, Tongji University; ${ }^{j}$ Shanghai Corps Hospital of the People's Armed Police Force; ${ }^{k}$ Chongming Branch of Xinhua Hospital Affiliated to Shanghai Jiao Tong University School of Medicine; ' Nanxiang Hospital, Jiading District, Shanghai; ${ }^{m}$ Shanghai Tenth People's Hospital, Ultrasound Research and Education Institute, School of Meicine, Tongji University; ${ }^{n}$ Longhua Hospital Affiliated to Shanghai University of Traditional Chinese Medicine; ${ }^{o}$ Jiading District Central Hospital Affiliated to Shanghai Health Medical College; ${ }^{p}$ Shanghai Tenth People's Hospital, Ultrasound Research and Education Institute, School of Meicine, Tongji University; ${ }^{q}$ Dahua Hospital, Xuhui District, Shanghai; ${ }^{r}$ Ruijin Hospital Affiliated to Shanghai Jiao Tong University School of Medicine; ${ }^{s}$ Changzheng Hospital Affiliated to Naval Medical University, Shanghai International Medical Center; ${ }^{t}$ Shanghai Baoshan District Hospital of Integrated Traditional Chinese and Western Medicine.

\section{References}

[1] Yazar FM, Baykara M, Karaağaç M, Bülbüloğlu E. The role of conventional ultrasonography in the evaluation of antrum wall thickness in obese patients. Obes Surg 2016; 26:2995-3000.

[2] Shen L, Zhou C, Liu L, Zhang L, Lu D, Cai J, et al. Application of oral contrast trans-abdominal ultrasonography for initial screening of gastric cancer in rural areas of China. Dig Liver Dis 2017; 49:918923.

[3] Li S, Wang XH, Wang Yi. Current status and prospect of ultrasonic diagnosis of gastric diseases in China. Chinese Journal of Medical Ultrasound (Electronic Edition) 2016; 13:401-405.

[4] Kimmey MB, Martin RW, Haggitt RC, Wang KY, Franklin DW, Silverstein FE. Histologic correlates of gastrointestinal ultrasound images. Gastroenterology 1989; 96(2 Pt 1):433-441.

[5] Liu Z, Guo J, Wang S, Zhao Y, Li J, Ren W, et al. Evaluation of transabdominal ultrasound after oral administration of an echoic cellulose-based gastric ultrasound contrast agent for gastric cancer. BMC Cancer 2015; 15:932.

[6] Ajay K. Singh. Gastrointestinal imaging. Sun HL, trans. Beijing: China Science and Technology Press, 2018:59-61.

[7] Jane Bates. Required Reading of Abdominal Ultrasound. Zhang JX, Lv K, trans. Beijing: People's Military Medical Press, 2013:183-190, 206-208.

[8] Leopold GR, Asher WM. Deleterious effects of gastrointestinal contrast material on abdominal echography. Radiology 1971; 98:637640.

[9] Bluth EI, Merritt CR, Sullivan MA. Ultrasonic evaluation of the stomach, small bowel, and colon. Radiology 1979; 133:677-680.

[10] Frucht H, Doppman JL, Norton JA, Miller DL, Dwyer AJ, Frank JA, et al. Gastrinomas: comparison of MR imaging with CT, angiography, and US. Radiology 1989; 171:713-717.

[11] MacFarlane PI, Miller V. Comparison of barium swallow and ultrasound in diagnosis of gastro-oesophageal reflux. Br Med J (Clin Res Ed) 1985; 291:411.

[12] Carré IJ. Comparison of barium swallow and ultrasound in diagnosis of gastro-oesophageal reflux in children. Br Med J (Clin Res Ed). 1985; 291:606-607.

[13] Guo XZ, The application of gastric window -85 ultrasound imaging agent in the diagnosis of gastric diseases Chin J Med 1988,68:288.

[14] Lund PJ, Fritz TA, Unger EC, Hunt RK, Fuller E. Cellulose as a gastrointestinal US contrast agent. Radiology 1992; 185:783-788.

[15] Lev-Toaff AS, Langer JE, Rubin DL, Zelch JV, Chong WK, Barone $\mathrm{AE}$, et al. Safety and efficacy of a new oral contrast agent for sonography: a phase II trial. AJR Am J Roentgenol 1999; 173:431436.

[16] Shiyan L, Pintong H, Zongmin W, Fuguang H, Zhiqiang Z, Yan Y, et al. The relationship between enhanced intensity and microvessel density of gastric carcinoma using double contrast-enhanced ultrasonography. Ultrasound Med Biol 2009; 35:1086-1091.

[17] Zhang JQ, Huang HM, Yang YH. The value of oral SonoVue in the diagnosis of gastric contrast-enhanced ultrasound Journal of the 
Second Military Medical University 2007; 28: 863-866.

[18] Liu Z, Ren W, Guo J, Zhao Y, Sun S, Li Y, et al. Preliminary opinion on assessment categories of stomach ultrasound report and data system (Su-RADS). Gastric Cancer 2018; 21: 879-888.

[19] Guo XZ, Zhang W. Clinical application of oral contrast-enhanced ultrasound in gastrointestinal patients Chinese Journal of Medical Ultrasound (Electronic Edition) 2010; 7: 334-365.

[20] Chen MH. Ultrasonography of digestive diseases Beijing: Beijing Press, 2003:295.

[21] Cao HG, Wang JR. Ultrasonography in the abdomen. Beijing: People's Medical Publishing House, 1994:307-375.

[22] Liu ZG, Guo XG. Standardized operation of upper gastrointestinal endoscopy. Journal of Clinical Gastroenterology 2008; 20: 197-201. [in Chinese]

[23] Lu WM. Ultrasonography in Clinical Gastrointestinal Diseases. Xi 'an: Fourth Military Medical University Press, 2004:29-38.

[24] Vivien Gibbs, David Cole, Antonio Sassano. Essential Reading of Ultrasonic Physics. Dai Q, Meng H, trans. Beijing: People's Military Medical Press, 2013:28-52.

[25] Jia Ziniren. Introduction to Ultrasound Equipment. Zhu Q, trans. Beijing: Science Press, 2019: 83-94.

[26] Song JL, Zhang JQ, Zhao HJ, et al. Values of non-vascular contrastenhanced ultrasound in guiding percutaneous drainage of upper gastrointestinal fistulas. Journal of Second Military Medical University 2010; 31: 753-755. [in Chinese]

[27] Mo JZ, Yuan YZ, Zou DW. Inhibitory and functional dysfunction of the digestive system. Shanghai: Shanghai Science and Technology Press, 2005:5-8.

[28] Joharjy IA, Mustafa MA, Zaidi AJ. Fluid-aided sonography of the stomach and duodenum in the diagnosis of peptic ulcer disease in adult patients. Ultrasound Med 1990; 9:77-84.

[29] Yang BW, Han H. Double contrast-enhanced ultrasonography in diagnosis of preoperative t staging of gastric cancer: a meta-analysis. Chinese Journal of Medical Imaging 2019; 27: 225-229. [in Chinese]

[30] Li T, Lu M, Song J, Wu P, Cheng X, Zhang Z. Improvement to ultrasonographical differential diagnosis of gastric lesions: The value of contrast enhanced sonography with gastric distention. PLoS One 2017;12: e0182332.

[31] P.E.S. Palmer. Ultrasound diagnosis manual. Zhang QP, trans. Beijing: People's Medical Publishing House, 1997:29-58.

[32] Worlicek H, Dunz D, Engelhard K. Ultrasonic examination of the wall of the fluid-filled stomach. J Clin Ultrasound 1989; 17: 5-14.

[33] Shang KZ, Chen JR. Gastroenterography principle and diagnosis. Shanghai: Shanghai Science and Technology Press, 1995, 175-179.

[34] Xu ZZ, Wang Y. Medical Ultrasound Terms Manual. Shanghai: Shanghai Science and Technology Press, 2009:49-52.

[35] Shou WD, Yan S. Medical Ultrasound Technical Dictionary. Shanghai: Shanghai Jiao Tong University Press, 1990:62-77.

[36] Berthold Block. Introduction and advanced guidelines for abdominal ultrasound. Wang WP, trans. 3 Ed. Tianjin: Tianjin Science and Technology Translation and Publishing Co., Ltd., 2019: 3-8.

[37] Feng LZ. Concise type B ultrasound diagnostics. Beijing: Beijing science and technology press, 1987:149-153.

[38] Hasegawa Y. Introduction to digestive tract ultrasound, Zhao H, trans. Beijing: Science press, 2018:11-22.

[39] Shang KZ. Radiological image of digestive system in China. Beijing: People's Medical Publishing House, 2002:3-10.

[40] Hameed S, Caro-Domínguez P, Daneman A, Zani-Ruttenstock E, Zani A, Navarro OM. The role of sonography in differentiating congenital intrinsic duodenal anomalies from midgut malrotation: emphasizing the new signs of duodenal and gastric wall thickening and hyperechogenicity. Pediatr Radiol 2020; 50:673-683.

[41] Giovanni Maconi, Gabriele Bianchi Porro. Gastrointestinal ultrasonography. Zhou ZY, Liu GJ, trans. 2nd Ed. Beijing: People's Medical Publishing House, 2018: 1-192.

[42] Chiu PWY, Uedo N, Singh R, Gotoda T, Ng EKW, Yao K, et al. An Asian consensus on standards of diagnostic upper endoscopy for neoplasia. Gut 2019; 68:186-197.

[43] Hosui Tozo. Standard gastroscopy. Wang X, Li YJ, Zhou JP, trans. Shenyang: Liaoning Science and Technology Press, 2013:68-75.

[44] Rose de Bruyn. Required reading of pediatric ultrasound. Huang PT, Chen CC, You XD, trans. 2nd Ed. Beijing: People's Military Medical Press, 2012:142-157.

[45] Du QJ, Cui LG. Ultrasonography memorandum of normal value measurement. Beijing: People's Military Medical Press, 2014:27.

[46] Pediatric ultrasound guidelines in China. Beijing: People's Medical Publishing House, 2018: 231-233.

[47] Shanghai Ultrasonic Quality Control Manual. 2006:42-45.

[48] Zhang Q, Chen ZY, Chen CD, Liu T, Tang XW, Ren YT, et al. Training in early gastric cancer diagnosis improves the detection rate of early gastric cancer: an observational study in China. Medicine (Baltimore) 2015; 94: e384. [in Chinese] 


\section{Appendix 1. Template of Normal Gastric Ultrasound Report (Recommended Version)}

Name of oral contrast agent:

Oral contrast medium dose: $\mathrm{ml}$

Scanning position: $\square$ standing $\square$ sitting $\square$ supine $\square$ right side decubitus

$\square$ left side decubitus $\square$ other

\begin{tabular}{|c|c|c|c|c|c|c|c|}
\hline \multirow{2}{*}{ Inspect area } & Cardia & Gastric fundus & Gastric body & Gastric Angle & Gastric antrum & Helicobacter tube & Duodenal bulb \\
\cline { 2 - 7 } & $\sqrt{ }$ & $\sqrt{ }$ & $\sqrt{ }$ & $\sqrt{ }$ & $\sqrt{ }$ & $\sqrt{ }$ \\
\hline $\begin{array}{c}\text { The quality of } \\
\text { imaging }\end{array}$ & $\begin{array}{c}\text { Ideal/good/not } \\
\text { good }\end{array}$ & $\begin{array}{c}\text { Ideal/good/not } \\
\text { good }\end{array}$ & $\begin{array}{c}\text { Ideal/good/not } \\
\text { good }\end{array}$ & $\begin{array}{c}\text { Ideal/good/not } \\
\text { good }\end{array}$ & $\begin{array}{c}\text { Ideal/good/not } \\
\text { good }\end{array}$ & $\begin{array}{c}\text { Ideal/good/not } \\
\text { good }\end{array}$ & $\begin{array}{c}\text { Ideal/good/not } \\
\text { good }\end{array}$ \\
\hline
\end{tabular}

\section{Ultrasonic description:}

Cardia: No obvious abnormal changes such as reflux.

Gastric fundus: No obvious abnormal changes.

Gastric body: Gastric wall thickness $\mathrm{mm}$, no obvious abnormal changes.

Gastric Angle: No obvious abnormal changes.

Gastric antrum: No obvious abnormal changes.

Pylorus: Inner diameter $\mathrm{mm}$, no obvious abnormal changes.

Duodenal bulb: No obvious abnormal changes.

Lower gastric boundary :( $\square$ Above the umbilicus $\square$ Below the umbilicus $\mathrm{cm})$.

Gastric filling and emptying: no obvious abnormalities observed.

\section{Ultrasonic result:}

\section{Examiner:}

Inspection date and time:

\section{Remark:}

1. Gastric ultrasound examination is one of the screening methods for gastric diseases, and gastroendoscopic gastric mucosal biopsy should be performed in time if necessary.

2. This report is for clinicians' reference only. 